\title{
Identification of SRGt 062340.2-265715 as a bright, strongly variable, novalike cataclysmic variable
}

\author{
Axel Schwope $^{1}$, David A.H. Buckley ${ }^{2,3}$, Adela Kawka ${ }^{4}$, Ole König ${ }^{5}$, Alexander Lutovinov ${ }^{6}$, Chandreyee Maitra ${ }^{7}$, Ilya \\ Mereminskiy $^{6}$, James Miller-Jones ${ }^{4}$, Manuel Pichardo Marcano ${ }^{8}$, Arne Rau ${ }^{7}$, Andrei Semena ${ }^{6}$, Lee J. Townsend ${ }^{2}$, and \\ Jörn Wilms ${ }^{5}$ \\ ${ }^{1}$ Leibniz-Institut für Astrophysik Potsdam (AIP), An der Sternwarte 16, 14482 Potsdam, Germany \\ e-mail: aschwope@aip.de \\ 2 South African Astronomical Observatory, P.O. Box 9, Observatory, 7935, Cape Town, South Africa \\ 3 Department of Astronomy, University of Cape Town, Private Bag X3, Rondebosch 7701, South Africa \\ 4 International Centre for Radio Astronomy Research, Curtin University, GPO Box U1987, Perth, WA 6845, Australia \\ 5 Dr. Karl Remeis-Sternwarte \& Erlangen Centre for Astroparticle Physics, Friedrich-Alexander-Universität Erlangen-Nürnberg, \\ Sternwartstr. 7, 96049 Bamberg, Germany \\ 6 Space Research Institute (IKI) of Russian Academy of Sciences, Profsoyuznaya ul 84/32, 117997 Moscow, Russian Federation \\ 7 Max-Planck-Institut für extraterrestrische Physik, Gießenbachstraße, 85748 Garching, Germany \\ 8 Department of Physics and Astronomy, Texas Tech University, USA
}

June 29, 2021

\begin{abstract}
We report the identification and follow-up of the transient SRGt 062340.2-265715 detected with both instruments on board the Spektrum-Roentgen-Gamma mission. Optical spectroscopy of the $G=12.5$ counterpart firmly classifies the object as a novalike cataclysmic variable (CV) at a distance of $495 \mathrm{pc}$. A highly significant TESS period of 3.941 hours, tentatively identified with the orbital period of the binary, could not be found when the object was reobserved with TESS two years later. The newer high-cadence TESS data revealed quasi-periodic oscillations around $25 \mathrm{~min}$, while ground-based photometry indicated periodic variability at 32 min. Located in very sparsely populated regions of color-magnitude diagrams involving X-ray and optical magnitudes and colors, the new object could be an X-ray underluminous magnetic CV, an intermediate polar, or an overluminous nonmagnetic CV. The lack of uniquely identified spin and orbital periods prevents a final classification. The site of X-ray production in the system, $L_{\mathrm{X}, \mathrm{bol}}=$ $4.8 \times 10^{32} \mathrm{erg} \mathrm{s}^{-1}$, remains to be understood given its high variability on long and short timescales.
\end{abstract}

Key words. stars: cataclysmic variables - X-rays: stars - stars: individual: SRGt 062340.2-265715

\section{Introduction}

In the course of the second all-sky survey, on 2020 October 12 , the two instruments on board the Spektrum-Roentgen-Gamma mission (SRG, Sunyaev et al. 2021) detected a bright transient at galactic coordinates $l^{I I}=234 \mathrm{~d} 7, b^{I I}=-17.6$. The comparison of data obtained during the first and second all-sky surveys with the two instruments on board the mission, the Mikhail Pavlinsky

$\checkmark$ ART-XC (Pavlinsky et al. 2021) and eROSITA (Predehl et al. 2021), revealed an increase in X-ray flux of about a factor 2, which triggered some fast-turnaround follow-up observations.

An initial announcement of the source was published by Schwope et al. (2020) and some analysis of the TESS data was reported by Pichardo-Marcano (2020). In this paper we describe the initial results in more detail, together with follow-up spectroscopy and photometry from the ground and from space. The transient was found to be coincident with cataloged ROSAT, Swift/XRT, and Gaia sources (1RXS J062339.8-265744 and 2SXPS J062339.9-265751) and the optical transient ZTF19aaabzuh, respectively. It was mentioned earlier as a cataclysmic variable by Denisenko on his webpage ${ }^{1}$, but no further supporting information was available.
The X-ray and optical observations uniquely identify the transient with a nova-like cataclysmic variable $(\mathrm{CV})$. CVs are close binaries with white dwarfs that accrete from a Rochelobe filling star. In approximate terms, they may physically be divided into magnetic and nonmagnetic objects (disk accretion vs. quasi-radial accretion), and phenomenologically, they are often sorted into dwarf novae (DN, which are nonmagnetic diskaccreting CVs that may become unstable and cause the DN outburst) and so-called novalikes. Historically, both magnetic and nonmagnetic CVs were sorted into this last class. The nonmagnetic disk-accreting novalikes (often referred to as UX UMas, although the VY Scl, Z Cam, and SW Sex objects and possibly other subtypes may also fall in this class) are objects with high-accretion rates whose disks are permanently found in the hot state so that no dwarf nova outbursts are launched. CVs of all subtypes were expected to be found in large number through systematic spectroscopic follow-up of point-like sources serendipitously discovered in the ongoing all-sky surveys with $e$ ROSITA (Schwope 2012). The case considered here is unusual through its very high optical brightness and its high and strongly variable $\mathrm{X}$-ray brightness in the hard $S R G$ band as well, which allowed its discovery with both instruments on the spacecraft.

\footnotetext{
${ }^{1}$ http://scan.sai.msu.ru/ denis/VarDDE.html
} 


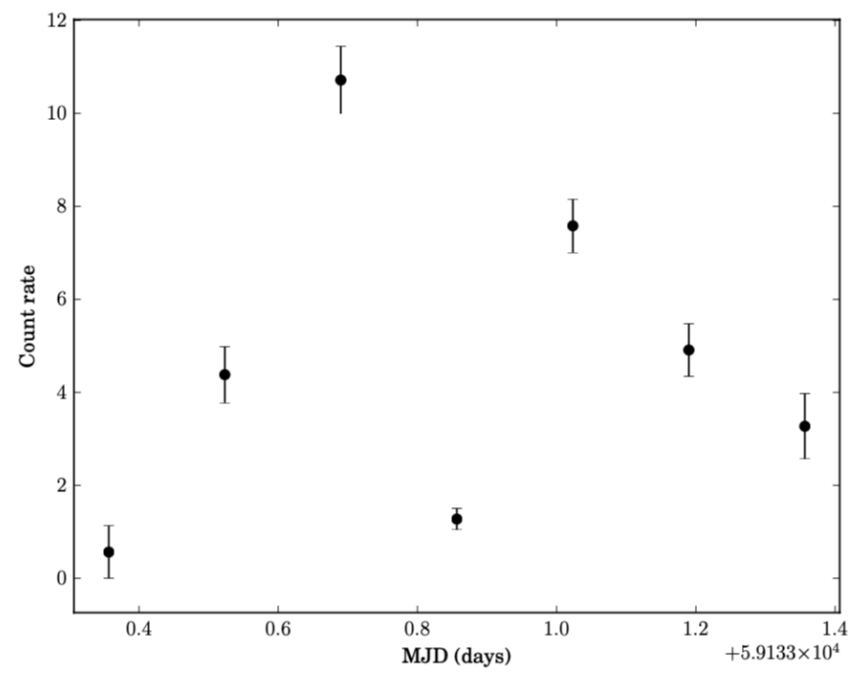

Fig. 1. X-ray light curve of SRGt062340.2-265715 obtained for eRASS2 $(0.2-10 \mathrm{keV})$. Each data point represents the mean rate of one individual scan over the source position.

\section{Observations and analysis}

\section{1. $S R G$}

The transient of 2020 October 12 was observed at the eROSITA position $\alpha_{\mathrm{J} 2000.0}=06^{\mathrm{h}} 23^{\mathrm{m}} 40 \mathrm{~s} 2(95.91754), \delta_{\mathrm{J} 2000.0}=$ $-26^{\circ} 57^{\prime} 15^{\prime \prime}(-26.96425)$ with a positional uncertainty of 1 '.6 (0'.6 statistical, 1'.5 systematic). The Mikhail Pavlinsky ART$\mathrm{XC}$ telescope detected the source at the position of $\alpha_{\mathrm{J} 2000.0}=$ $06^{\mathrm{h}} 23^{\mathrm{m}} 39.7, \delta_{\mathrm{J} 2000.0}=-26^{\circ} 57^{\prime} 53^{\prime \prime}$ with an uncertainty of $15^{\prime \prime}$ (90\%).

During the second $S R G$ survey, the object had a total exposure time of $85 \mathrm{~s}$ (an effective exposure time of $25 \mathrm{~s}$ ) with ART-XC and was discovered at a mean flux of $1.3_{-0.3}^{+0.4} \times$ $10^{-11} \mathrm{erg} \mathrm{cm}^{-2} \mathrm{~s}^{-1}$ in the $4-12 \mathrm{keV}$ energy band. It was below the detection threshold during the first survey, when it had an effective exposure time of $21 \mathrm{~s}$, corresponding to an upper limit for the 4-12 keV flux of $F<10^{-11} \mathrm{erg} \mathrm{cm}^{-2} \mathrm{~s}^{-1}$. With eROSITA, the source was detected in both surveys, in eRASS1 (2020 April 8 ), where it received a total exposure time of $228 \mathrm{~s}$, with a mean count rate of $1.38 \pm 0.11 \mathrm{~s}^{-1}$, and in eRASS2 (2020 October 12) with a mean rate of $5.84 \pm 0.24 \mathrm{~s}^{-1}$ for a total exposure of $208 \mathrm{~s}$.

More than 1200 photons were collected during eRASS2, which allowed generating the eRASS2 light curve that is displayed in Fig. 1. Circular regions with radii of $75^{\prime \prime}$ and $100^{\prime \prime}$ were used for the source and background extraction, respectively. SRGt 062340.2-265715 was observed during seven erodays $^{2}$ and showed variability between $10.7 \pm 0.7 \mathrm{~s}^{-1}$ and $1.3 \pm$ $0.2 \mathrm{~s}^{-1}$. Most of the photons registered with ART-XC were registered during the fourth and fifth eroday (see Fig. 1).

The combined spectrum from the two instruments on board SRG is displayed in Fig. 2. It was fit using XSPEC version 12.11 with a one-component emission model, a thermal plasma absorbed by cold interstellar matter. A scaling factor between the two spectral groups was also fit that accounts (a) for calibration uncertainties between the two involved instruments and (b) for the fact that the data were not taken strictly si-

\footnotetext{
${ }_{2}$ An eroday lasts 4 hours and corresponds to the revolution of the spacecraft around the scan axis. The exposure for a given sky position is different for each eroday and may last up to $40 \mathrm{~s}$ dependent on the off-axis angle
}

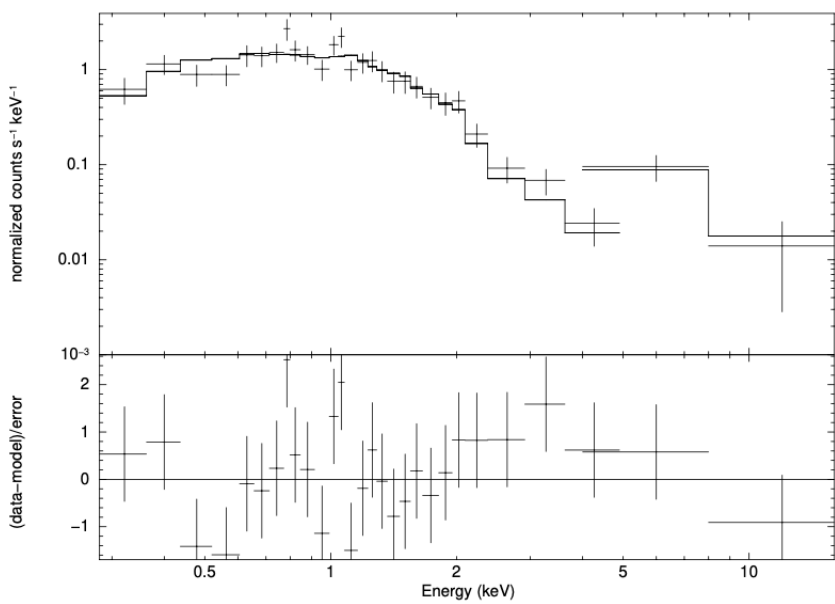

Fig. 2. Mean combined spectrum of ART X-C and eROSITA of SRGt 062340.2-265715 obtained during the second survey with SRG with the best-fitting thermal model spectrum. The lower panel shows the residuals in units of the error per spectral bin.

multaneously, which is important given the high variability of the source. The C-statistic was used for fitting. The best-fit value found was 180 for 230 bins. This provides a temperature $k T=5.7_{-1.6}^{+2.6} \mathrm{keV}$ (errors given for a $90 \%$ confidence interval) and fluxes of $F_{\text {eRO }}(0.5-2.0 \mathrm{keV})=4.3_{-0.4}^{+0.3} \times 10^{-12}$ $\operatorname{erg~cm}{ }^{-2} \mathrm{~s}^{-1}, F_{\mathrm{eRO}}(0.5-10 \mathrm{keV})=1.2_{-0.4}^{+0.5} \times 10^{-11} \mathrm{erg} \mathrm{cm}^{-2} \mathrm{~s}^{-1}$, and $F_{\text {ART }}(4-12 \mathrm{keV})=2.0_{-0.3}^{+0.14} \times 10^{-11} \mathrm{erg} \mathrm{cm}^{-2} \mathrm{~s}^{-1}$. The column density was determined using the TBabs command (abundance set to wilms), but was not found to be well constrained. It was therefore fixed at the Galactic column density in this direction of $\mathrm{N}_{\mathrm{H}}=4.08 \times 10^{20} \mathrm{~cm}^{-2}$ (HI4PI Collaboration et al. 2016). Recent $3 \mathrm{D}$ dust maps (e.g., Green et al. 2019) do not provide better constraints due to rather large errors, $E(g-r)=0.03 \pm 0.02$. The implied column density is compatible with that inferred from X-rays. The same model applied to the eRASS1 data with a fixed amount of interstellar absorption gave a lower temperature $k T=2.0_{-0.6}^{+3.4} \mathrm{keV}$ at a flux of $F_{\mathrm{X}}(0.5-2.0 \mathrm{keV})=(8.4 \pm 1.6) \times 10^{-13} \mathrm{erg} \mathrm{cm}^{-2} \mathrm{~s}^{-1}$. The bolometric flux of the best-fitting model during the high state in October 2020 was $F_{\mathrm{X}}=1.6 \times 10^{-11} \mathrm{erg} \mathrm{cm}^{-2} \mathrm{~s}^{-1}$ (eROSITA value).

\subsection{Gaia}

Gaia EDR3 lists an object (source_ID 2899766827964264192) at a distance of 2.44 from the eRASS2 catalog position of the transient at position RA $=95.91672681385$, DEC $=$ -26.96405593108 . A finding chart indicating the eRASS2 Xray position and all Gaia sources in its vicinity is reproduced in Fig. 3. It has a parallax of $\pi=1.9749 \pm 0.0192$ mas and photometric properties $G=12.436 \pm 0.005 \mathrm{mag}$ and $B p-R p=0.023$. Following Bailer-Jones et al. (2021), the median geometric distance to SRGt $062340.2-265715$ is $495.5 \pm 4 \mathrm{pc}$, which gives an absolute magnitude $M(G)=3.96$.

\subsection{Archival $X$-ray observations}

The position of SRGt $062340.2-265715$ was covered previously by past (ROSAT) and current (XMM-Newton and Swift/XRT) Xray missions. The corresponding information is available via the ESA-supported high-energy light-curve generator HILIGT (up- 
Table 1. Archival X-ray observations of SRGt 062340.2-265715 as derived from the ESA upper limit server HILIGT

\begin{tabular}{lcrlll}
\hline \hline Mission & Date & Exp time & Flux $^{a}$ & Flux $^{a}$ & Flux $^{a}$ \\
& & $(\mathrm{~s})$ & $0.2-2 \mathrm{keV}$ & $2-12 \mathrm{keV}$ & $0.2-12 \mathrm{keV}$ \\
\hline ROSAT-Survey & $1990-09-1900: 47: 17$ & 661.5 & $2.1 \pm 0.2$ & & \\
XMM-Newton slew & $2003-04-01$ & 5.6 & $2.3 \pm 0.8$ & $<28.2$ & $6.4 \pm 2.0$ \\
XMM-Newton slew & $2006-10-02$ & 9.9 & $5.0 \pm 0.8$ & $11.6 \pm 3.3$ & $12.4 \pm 1.8$ \\
Swift-XRT & $2008-07-17$ & 5558.3 & $1.7 \pm 0.1$ & $2.3 \pm 0.2$ & $3.8 \pm 0.2$ \\
\hline
\end{tabular}

${ }^{a}$ Absorbed flux in units of $10^{-12} \mathrm{erg} \mathrm{cm}^{-2} \mathrm{~s}^{-1}$. Count rate to flux conversion performed with a power-law spectral model of slope 2 and absorption $N_{\mathrm{H}}=3 \times 10^{20} \mathrm{~cm}^{-2}$.

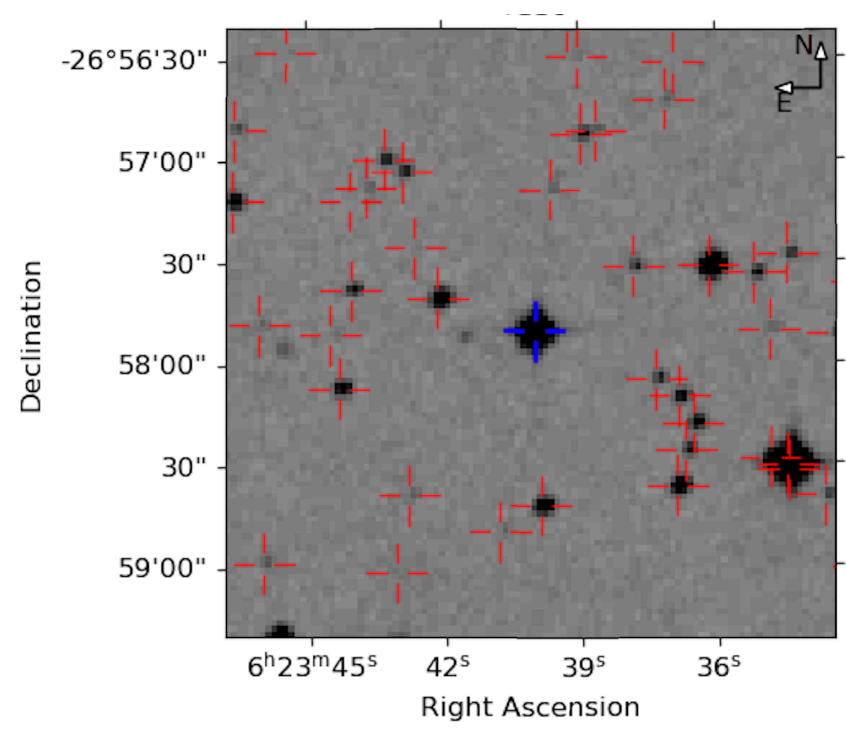

Fig. 3. Finding chart of the new transient $(3 \times 3$ arcmin). The background image is from the DSS, and the eROSITA position (eRASS2) is marked with a blue cross. All further Gaia objects in the field of view are marked with red crosses.

per limit server $)^{3}$ and listed in Tab. 1. Although HILIGT does not offer a thermal spectral model to convert count rates into fluxes, the given values are at least indicative. The flux in the soft band, $0.2-2.0 \mathrm{keV}$, common to all the missions, was found to be variable by at least a factor 2 .

\subsection{Initial follow-up spectroscopy with WiFeS and SALT}

The likely Gaia counterpart was selected for follow-up spectroscopy with the $2.3 \mathrm{~m}$ telescope of the Australian National University, which is equipped with the Wide Field Spectrograph (WiFeS; Dopita et al. 2010) and with the $10 \mathrm{~m}$ class Southern African Large Telescope (SALT; Buckley et al. 2006)), which is equipped with the High Resolution Spectrograph (HRS; Crause et al. 2014). WiFeS is a double-beam, image-slicing, integral-field spectrograph. Observations were performed on 2020 October 28.164 with the R3000 grating and an exposure of $300 \mathrm{~s}$. As the red arm of the instrument was not operating, only the blue wavelength range between 3500 and $5500 \AA$ is available. The WiFeS spectrum was reduced using the PyWiFeS reduction pipeline (Childress et al. 2014), which produced three-dimensional data. We then extracted backgroundsubtracted spectra from the slitlets that provided significant flux.

\footnotetext{
${ }^{3}$ http://xmmuls.esac.esa.int/hiligt/
}
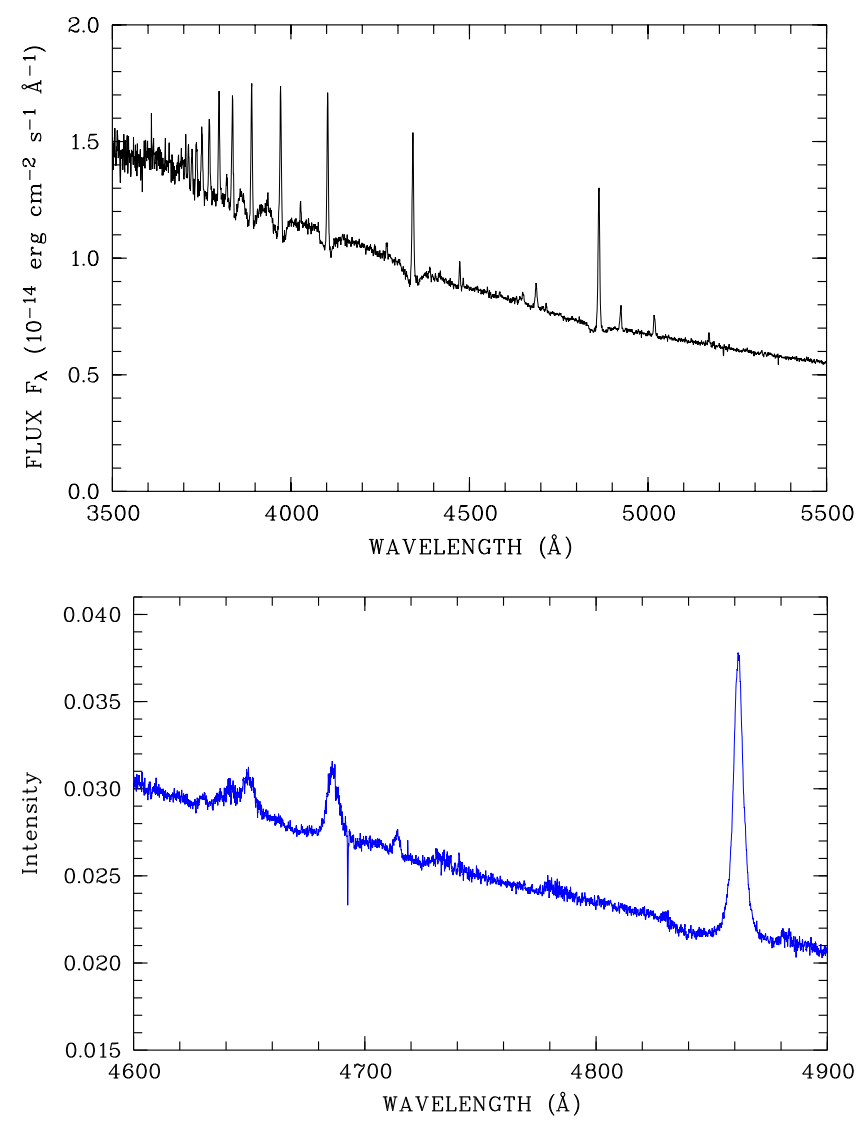

Fig. 4. ANU/WiFeS low-resolution and SALT/HRS high-resolution identification spectra of SRGt 062340.2-265715. The SALT spectrum only has arbitrary uncalibrated intensity units. There is high excitation

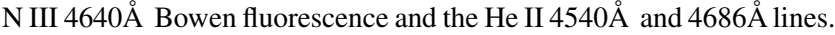

HRS is a dual-beam, fibre-fed èchelle spectrograph, covering 3800-8900 $\AA$. A 1200 s HRS observation was taken on 2020 October 31.9672 in low-resolution (LR; $R \sim 15,000$ ) mode. Initial reduction of the HRS spectrum was achieved using the PySALT package (Crawford et al. 2010), which includes overscan correction, bias subtraction, and gain correction. The spectrum was extracted using the HRS pipeline, based on MIDAS routines described in Kniazev et al. (2016). The ANU/WiFeS and SALT/HRS spectra are displayed in Fig. 4.

The object has a very blue continuum that increases to the short-wavelength cutoff at $3500 \AA$. The WiFeS spectrum reveals broad Balmer absorption lines whose centers are filled with intense emission lines. In addition to H-Balmer emission lines, the object displays He-emission lines, both neutral and ionized. 


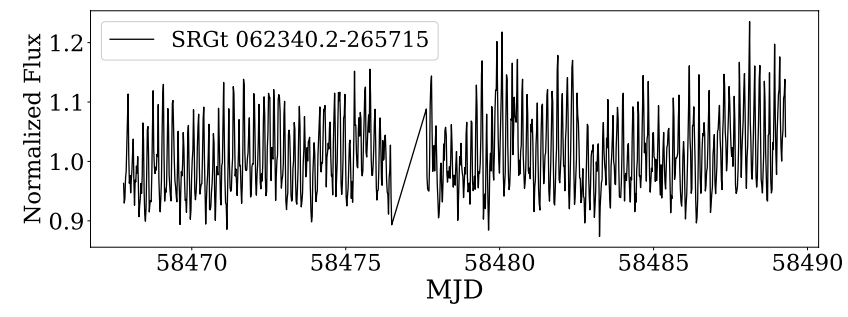

Fig. 5. TESS light curve for the SRGt 062340.2-265715. The normalized light curve was obtained by dividing the background-corrected source flux by its median value of 513.1 electrons $\mathrm{s}^{-1}$.

Through these early spectra, the object is robustly identified as a $\mathrm{CV}$. None of the spectra shows any sign of the donor star.

The typical full width at half maximum (FWHM) of the absorption lines corresponds to $3000 \mathrm{~km} \mathrm{~s}^{-1}$, and those of the Balmer emission lines correspond to $300-380 \mathrm{~km} \mathrm{~s}^{-1}$. The ionized helium line at $4686 \AA$ is detected, but appears to be rather weak. It has an equivalent width of $\sim-0.9 \AA$, an FWHM of $\sim 6 \AA\left(\sim 380 \mathrm{~km} \mathrm{~s}^{-1}\right)$, and the flux ratio to the Balmer emission line $\mathrm{H} \beta$ is $F(\mathrm{He}$ II $) / F(H \beta) \simeq 0.26$. $\mathrm{H} \beta$ has an equivalent width of $\sim-4.2 \AA$ and an FWHM of $\sim 5.5 \AA\left(\simeq 340 \mathrm{~km} \mathrm{~s}^{-1}\right)$. The HRS spectrum of the region around He II 4686 also uncovers the Bowen blend of $\mathrm{N}$ III lines. This feature is often encountered in bright low-mass X-ray binaries (LMXBs, e.g., Cornelisse et al. 2008), but also in the two types of magnetic CVs, the polars and the intermediate polars (e.g., Schachter et al. 1991; Harlaftis \& Horne 1999), indicating a strong UV/EUV source.

The $\mathrm{H} \beta$ emission line has a centroid of $4861.54 \AA$ in the SALT spectrum, whereas it was found at $4862.59 \AA$ in the WiFeS spectrum while being similarly broad ( $5.2 \AA$ vs. $5.5 \AA$ ). The difference in wavelength corresponds to a velocity difference of $65 \mathrm{~km} \mathrm{~s}^{-1}$, illustrating the feasibility of a radial velocity study.

\subsection{TESS}

We analyzed data from the Transiting Exoplanet Survey Satellite (TESS, Ricker et al. 2014). TESS, using four CCD cameras, obtains continuous optical images of a rectangular field of $24^{\circ} \times 90^{\circ}$ for 27.4 days at a short cadence ( 30 minutes to 20 seconds).

The source was observed in sector 6 , which was observed at a 30-minute cadence between MJD 58467.79 to MJD 58489.54 (2018 December 15 to 2019 January 06). The source was also observed in sector 33 at a faster 2-minute cadence between MJD 59201.24 (2020 December 18) and MJD 59227.07 (2021 January 13$)$.

We began the analysis with the data obtained in sector 6 (30 min cadence) and used the Python package Lightkurve (v1) (Lightkurve Collaboration et al. 2018) to create the light curves from the full-frame images (FFI). From the FFI from which we extracted our light curves, we selected pixels around the coordinates of the target, and background or empty pixels with no known Gaia sources as our background and with a median flux that is lower than a given threshold. We also used the TESS light curve from the MIT Quick-Look Pipeline (QLP, Huang et al. 2020) and compared them to our light curves from the full-frame images using the median background-removal method. The results from the two programs agreed well. The final extracted light curve (30 min cadence), normalized to the median value of 513.1 electrons s ${ }^{-1}$, is displayed in Fig. 5. The light curve is flat to first order, but displays an apparent flickering that gives the

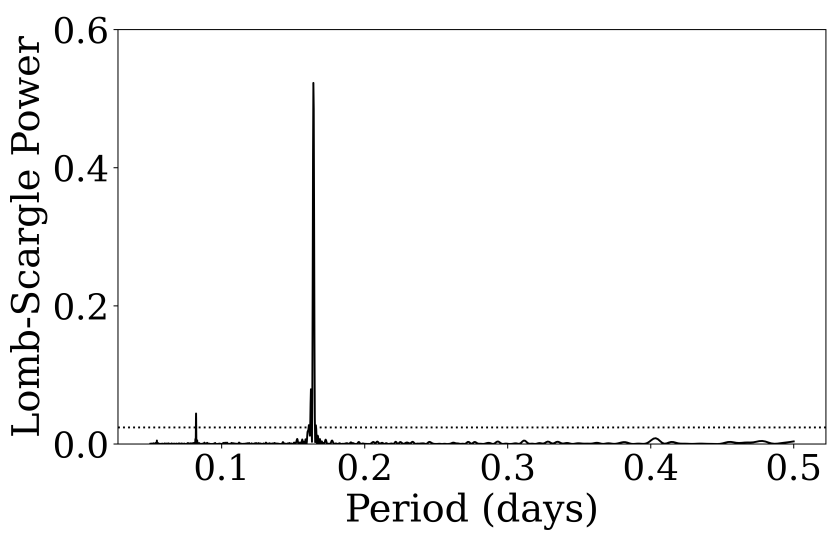

Fig. 6. Lomb-Scargle periodogram for SRGt 062340.2-265715, showing a clear isolated peak around 3.941 hours. The dotted line shows the periodogram level corresponding to a maximum peak false-alarm probability of $1 \%$ using the bootstrap method that simulates data at the same observation times to approximate the true distribution of peak maxima for the case without a periodic signal. The bootstrap method is performed with periods between 0.1 and 0.5 days and after normalizing by the residuals of the data around a constant reference model.

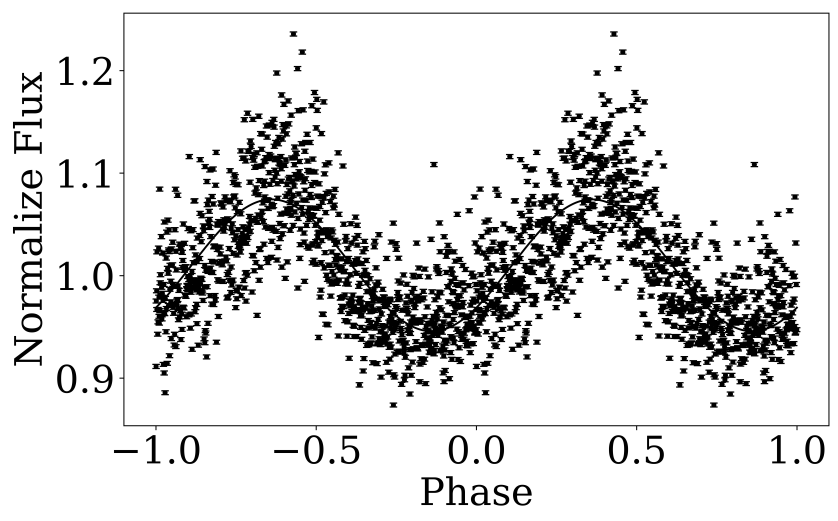

Fig. 7. TESS light curve for SRGt 062340.2-265715, folded on the 3.941-hour period. Data are shown with their error bars. The solid lines represent the best-fit sinusoid. Phase zero was chosen arbitrarily.

impression of a possible periodic behavior. We thus performed a periodogram analysis to search for any underlying periodicity.

We performed period searches using the Lomb-Scargle (LS, Lomb 1976; Scargle 1982) and the phase dispersion minimization technique (PDM, Stellingwerf 1978) as implemented in the Astropy and the PyAstronomy packages (Astropy Collaboration et al. 2018; Czesla et al. 2019). The LS-periodogram was then normalized by the residuals of the data around a constant reference model. The periodogram (Fig. 6) clearly shows an isolated peak at a period of $3.941 \pm 0.010 \mathrm{~h}$, the error being estimated from the width of the peak $(\sigma \sim 0.01 \mathrm{~h})$. We also calculated a falsealarm probability of $3.9 \times 10^{-158}$ using the method described in Baluev (2008).

In the PDM method, the light curve is divided into different phase bins, and the cost function, $\Theta=s^{2} / \sigma^{2}$, is minimized to choose the best period. Here $s$ is the phase bin variance and $\sigma$ is the total data variance. We divided the data into ten equidistant bins to calculate $\Theta$ and also found a period of 3.941 hours, which is fully consistent with the results obtained with the Lomb-Scargle periodogram. 


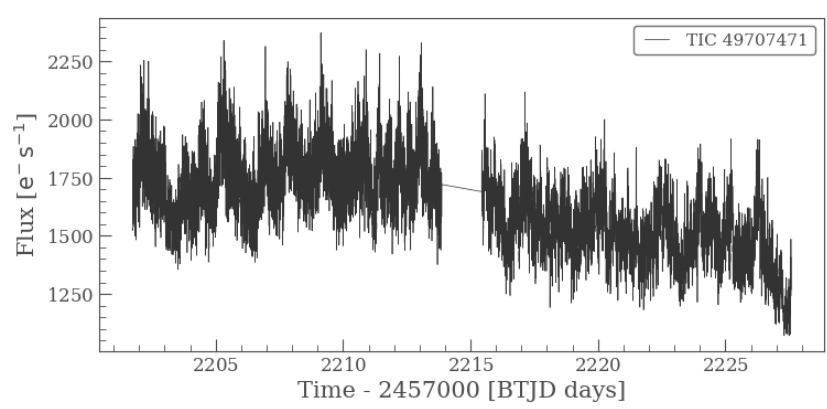

Fig. 8. TESS light curve for SRGt 062340.2-265715 obtained in sector 33 with a 2 min cadence.

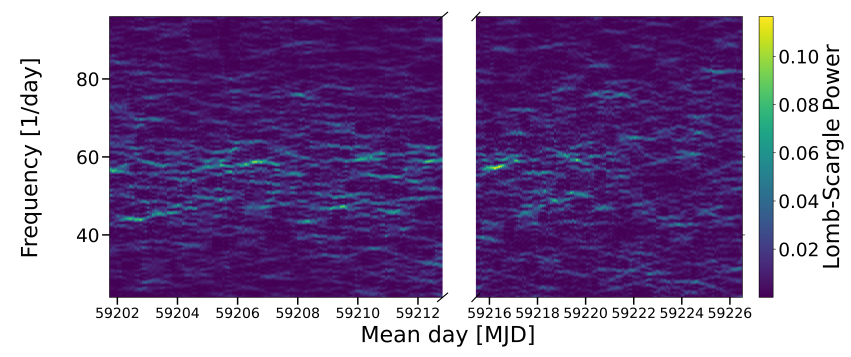

Fig. 9. Moving-window periodogram of the TESS 2-minute cadence data for a sliding window of one-day length and time steps of two hours. The $\mathrm{x}$-axis is the middle of the one-day window, and the color corresponds to the Lomb-Scargle power. The broken axis corresponds to the dates around the middle of the light curve that have some missing data.

The main result of the analysis of the 30-minute cadence data, the presence of a periodicity at about 3.9 hours, was reported very briefly already in ATEL 14222 by one of us (Pichardo-Marcano 2020). The more thorough analysis presented here confirms and refines the initial result. The light curve folded at the period of 3.941 hours is shown in Fig. 7. It is slightly skewed, with a more gradual increase and a steeper decrease. There is significant scatter at any given phase, which indicates further variability on shorter timescales. This could not be resolved with the low-cadence TESS data of sector 6 .

For the data obtained with a 2-minute cadence we used the Pre-search Data Conditioning Simple Aperture Photometry flux (PDCSAP), in which long-term trends have been removed using so-called cotrending basis vectors (CBVs, Smith et al. 2012), produced by the TESS Science Processing Operations Center (SPOC; Jenkins et al. 2016). The light curve of data from sector 33 is shown in the original time sequence in Fig. 8. On this occasion, the source appeared fundamentally different compared to the data obtained in sector 6 . At the beginning of the observations, the source was about 1.4 mag brighter than two years before, and at the end of this one-month interval only by about 0.9 mag. A Lomb-Scargle period search for the whole dataset from sector 33 no longer showed the $3.9 \mathrm{~h}$ modulation. We then selected shorter time intervals of one or two days length at the beginning, center, and end of the data train and searched for periodic behavior between 10 minutes and 5 hours. This revealed some power at $25 \mathrm{~min}$. To study the stability of this period, we calculated a dynamic periodogram using a time slice with a length of one day that was moved forward with a step size of 2 hours. For each slice, an LS periodogram was computed, and the results were arranged as the two-dimensional periodogram (LS power as a function of time) that we show in Fig. 9.

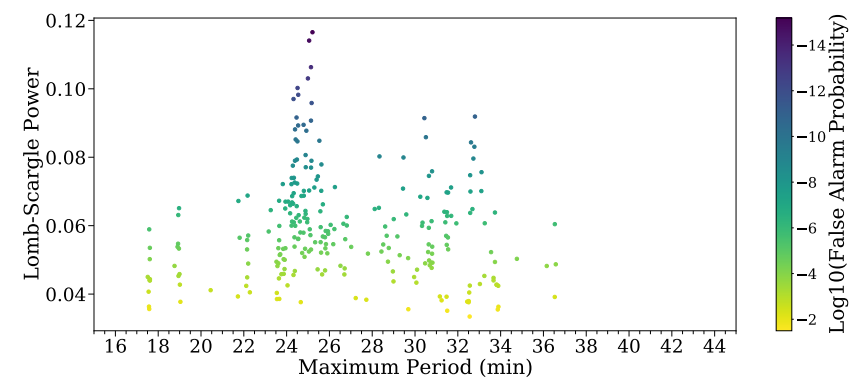

Fig. 10. For each one-day window we plot the maximum amplitude of the periodogram and its corresponding period. The color corresponds to the $\log _{10}$ of the FAP.

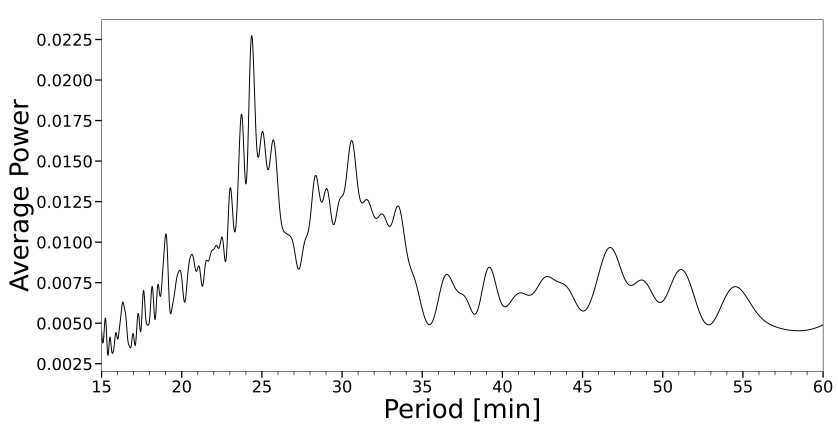

Fig. 11. Mean periodogram of sector 33 calculated by averaging all oneday periodograms of Fig. 9.

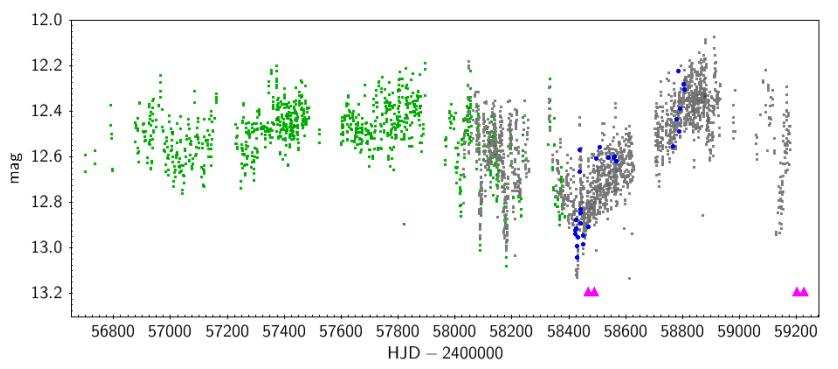

Fig. 12. ASASS-SN and ZTF photometric data for SRGt 062340.2-265715 (green symbols show ASAS-SN $V$, gray symbols show ASAS-SN $g$, and blue symbols represent ZTF $g$ ). The magenta triangles mark TESS observations with low and high cadence (the first and second pair identify TESS sectors 6 and 33, respectively).

Complementary views on the complex timing behavior of the source are given in Figs. 10 and 11, which show the falsealarm probabilities of the highest peak in each of the one-day periodograms and the mean periodogram of all the individual oneday periodograms. We found significant power centered around 25 min (frequency $57.6 \mathrm{~d}^{-1}$ ), but measured significant frequencies that jumped between 45 and 65 cycles per day on timescales of about one day. No significant period was found during the last quarter of the data set. The mean periodogram has a maximum power at $24.37 \mathrm{~min}$, and the most significant period in a one-day time slice is observed at $25.201 \mathrm{~min}$ with a false-alarm probability of $6.58 \times 10^{-16}$. 


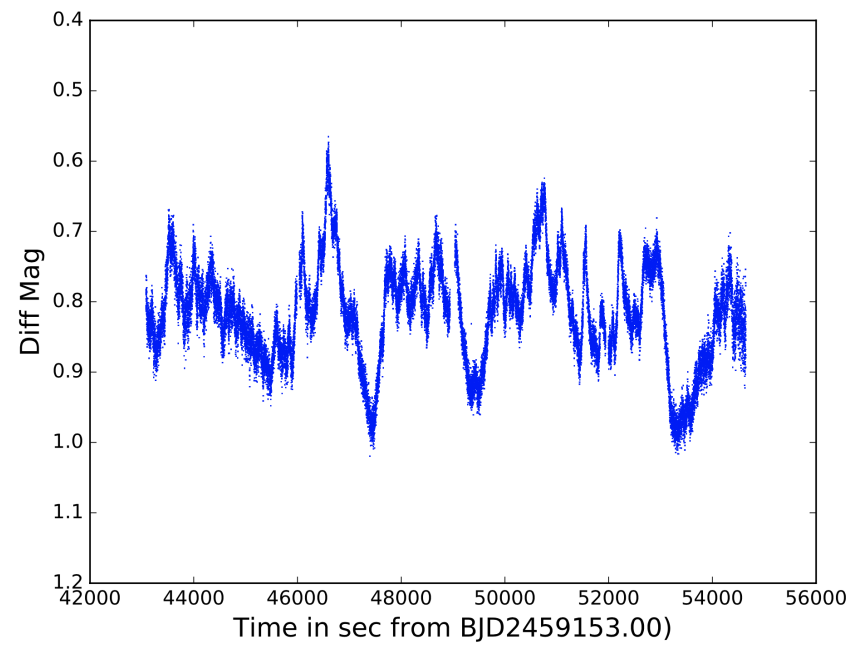

Fig. 13. Differential white-light optical light curve, spanning $3.2 \mathrm{~h}$, of SRGt $062340.2-265715$ obtained with $S A A O / S H O C$ at 0.3 s cadence. Time is in seconds since 00:00 UT on 1 November 2020.

\subsection{Archival photometry from CRTS, ZTF, ASAS-SN, and ATLAS}

Archival photometric information of the new transient is available from the Catalina Real-Time Transient survey (CRTS, Drake et al. 2009, 196 epochs), the Zwicky Transient Facility (ZTF, Masci et al. 2019, 55 epochs), the All-Sky Automated Survey for Supernovae (ASAS-SN, Shappee et al. 2014; Kochanek et al. 2017, 3350 epochs), and the Asteroid Terrestrial-impact Last Alert System (ATLAS, Tonry et al. 2018; Heinze et al. 2018, 34 epochs). The observations of these surveys cover the time intervals 2005 August 28 to 2010 April 19, 2018 November 1 to 2019 November 18, 2014 February 11 to 2020 November 24, and 2021 February 16 to 2021 March 16, respectively, and part of them are shown in Fig. 12 (omitting the CRTS and ATLAS data). ZTF data were taken in the $g$ and $r$ filters with only $g$-band data shown, and ASAS-SN data through $g$ and $V$ filters. All these data show extended phases (years) with little variability around a mean magnitude of about 12.5, with superposed scatter with an amplitude of 0.1 mag followed by dimmed phases with a larger short-term variability amplitude. These excursions toward lower brightness were observed twice, one at the end of the CRTS data train at MJD 56350, and the other was covered by both ZTF (partially) and ASAS-SN at MJD 58400. The minimum brightness of these fainter states is at around 13 mag. TESS observations in sector 6 revealing the 3.9 hour periodicity were obtained at the pronounced minimum. No automated survey data (e.g., ASAS-SN or ATLAS) are available at the time of writing when TESS observed the source again in sector 33 .

We searched for periodic behavior of the source in the ASAS-SN data. We selected $g$-band data obtained between HJD 2458455 and 2458866. During this 400-day interval with 1072 data points, the transient recovered from its faint state at $g=12.95$ to a bright state at $g=12.3$ with a constant gradient. The brightness displayed a scatter with a mean amplitude of 0.085 mag around the trend, while the mean magnitude error was only $0.01 \mathrm{mag}$. We used the LS period search method as implemented in ESO-MIDAS and found a period 3.9142 \pm 0.0011 hours, which is consistent with the value found in the TESS data at $2.5 \sigma$.

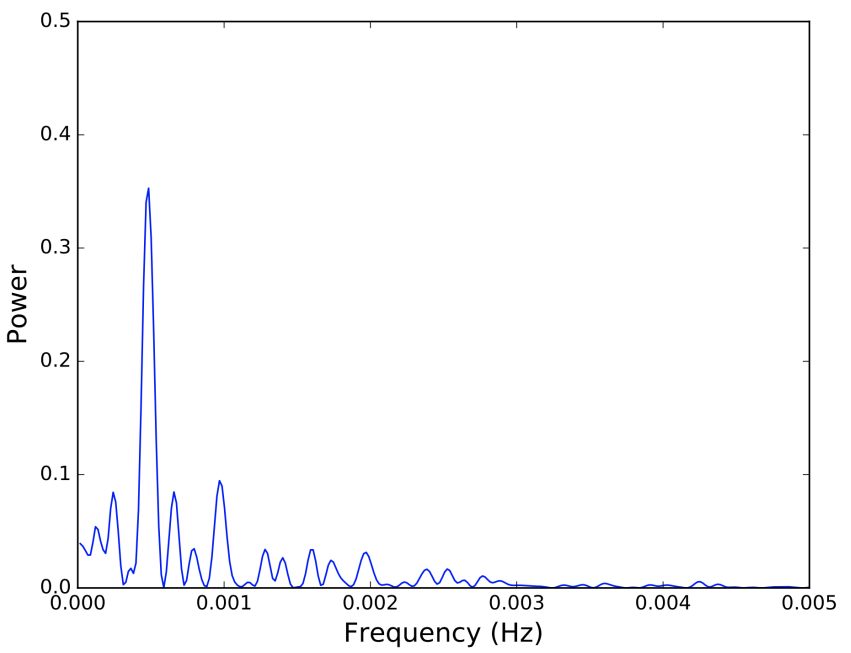

Fig. 14. Lomb-Scargle power spectrum of the light curve of SRGt 062340.2-265715 (shown in Fig 13) indicating a possible $\sim 2100$ s periodicity.

\section{7. $S A A O$ high-speed photometry}

Following its spectral identification (see subsection 2.4), we performed $3.2 \mathrm{~h}$ of high-speed photometry with the South African Astronomical Observatory $1.0 \mathrm{~m}$ telescope on 2020 November 1, beginning at 23:57:30 UTC. The Sutherland High speed Optical Camera (SHOC; Coppejans et al. 2013) CCD camera, which uses an Andor iXon888 frame transfer EM-CCD frame camera $(1024 \times 1024$ pixels $)$, was used. The observations were made without a filter (i.e., 'white light') with an exposure time of $0.3 \mathrm{~s}$.

The CCD images were reduced using the TEAPhot photometry reduction package (Bowman \& Holdsworth 2019) and included subtraction of median bias and flat-field correction using median-combined frames from exposures of the twilight sky. TEAPhot employs the method of adaptive elliptical aperture photometry, which was used to create calibrated science images and a differential light curve using the bright $(\mathrm{V}=12.1)$ reference star, UCAC2 20781392. The differential light curve is shown in Fig. 13.

The light curve shows strong flickering that is typical of CVs and is reminiscent of some intermediate polars that exhibit spinrelated periodic modulations. We therefore subjected the light curve to a Lomb-Scargle period analysis, probing frequencies as high as the Nyquist limit (0.6 s). Except for a strong peak at $0.480 \mathrm{mHz}$, there were no other significant peaks. The result is shown in Fig. 14. The strong peak corresponds to a period of $2080 \pm 100 \mathrm{~s}$ (34.7 min).

\section{Results and discussion}

We have analyzed space- and ground-based data of SRGt 062340.2-265715, an object that was initially detected as an X-ray transient with the two X-ray instruments on board SRG. Follow-up data, in particular the initial spectroscopy, uniquely identify the object as a $\mathrm{CV}$ and thus confirm Denisenko's previous classification. At $G=12.5 \mathrm{mag}$ the object is clearly one of the brighter examples of its class. An important question is to which, if any, CV family the object belongs.

The obtained spectra and the location of the object in the color-magnitude diagram (see Fig. 15) all strongly suggest that 


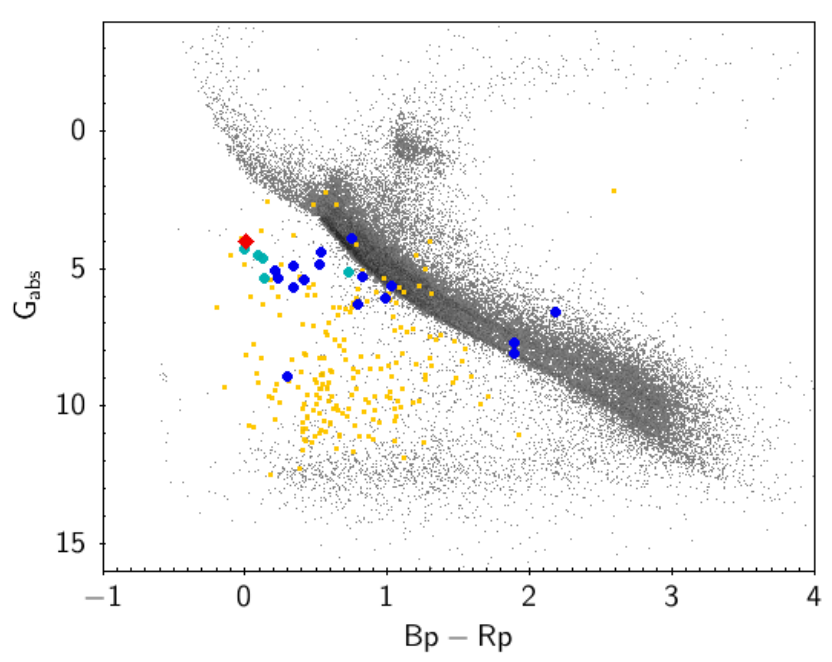

Fig. 15. Color-magnitude diagram for eRASS2 sources. Each gray dot is an eRASS2 source that is matched (within $8 \operatorname{arcsec}$ ) with a Gaia source within $500 \mathrm{pc}$ (distances by Bailer-Jones et al. 2018). In dark yellow we plot all CVs from the final Ritter \& Kolb catalogue, in green the nonmagnetic high-accretion rate UX UMas therein, and in blue the intermediate polars from Mukai's compilation. The new CV SRGt $062340.2-265715$ is identified by the red symbol.

it belongs to the novalike subclass. No dwarf nova outburst was recorded in the multi-year monitoring observations by CRTS.

The great majority of novalikes are found above the CV period gap. It is thus tempting to associate the 3.9-hour periodicity found in ASAS-SN and TESS data (sector 6) with the orbital period of the binary. The disappearance of this period in TESS sector 33 data is puzzling. It might be premature to associate the 3.9-hour periodicity with the orbital period , and alternatively, the photometric phenomenology might be completely changed in the much higher accretion state of TESS sector 33 data. Timeresolved spectroscopy is needed to uniquely identify the orbital period by tracing the absorption lines and the emission cores through an orbital cycle. The blue optical continuum and the absorption lines are thought to originate from an optically thick accretion disk. The narrow emission lines might be resolved into two components, one to be associated with the cooler outer parts of the disk and the other originating from the irradiated hemisphere of the donor star (see, e.g., Beuermann \& Thomas 1990; Hernandez et al. 2017, for templates), each with its own radial velocity curve.

The production sites of X-rays in nonmagnetic novalikes are debated. Originally, X-rays were assumed to originate from the boundary layer (BL) between the disk and the white dwarf with a blackbody-like spectral shape, but the predicted blackbodies were not found (Mauche \& Mukai 2002). The BL is nevertheless mostly regarded as the X-ray production site (Mukai 2017), but other locations such as shocked circumstellar material, the polar caps, a central corona, a partially obscured boundary layer, or advective hot flows are discussed (Zemko et al. 2014; Dobrotka et al. 2017; Balman 2020). The already observed high degree of X-ray variability on long and short timescales, that is, between eRASS surveys and between erodays, together with dedicated spectral observations may shed new light on this old question. If it belongs to the nonmagnetic novalikes, SRGt 062340.2-265715 likely does not belong to the VY Scl subclass because it does not show the typical anti-dwarf nova dimmings by $\sim 4$ mag.

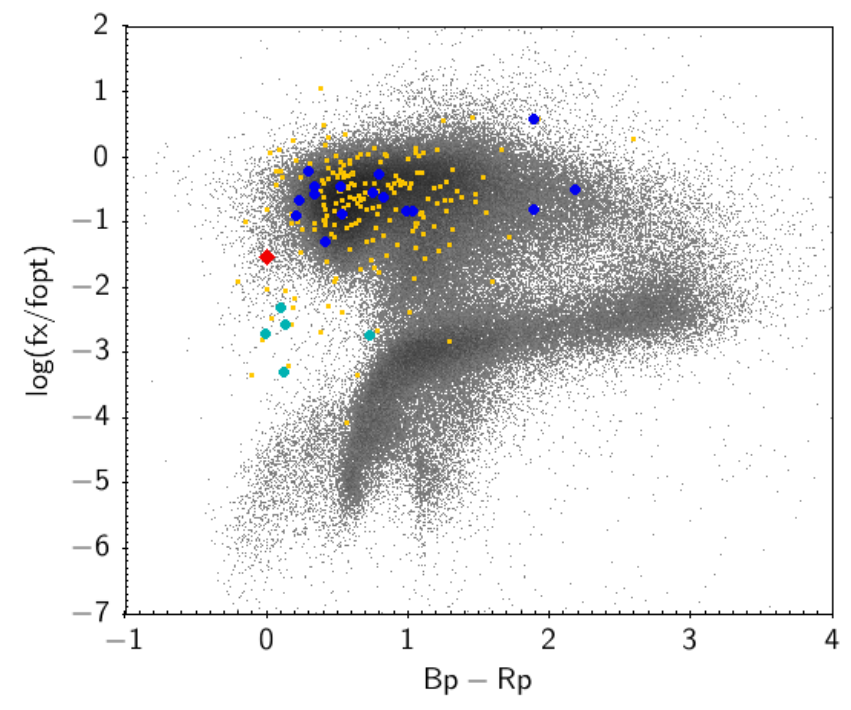

Fig. 16. X-ray to optical color-color diagram. Gray background objects are all eRASS2 sources that match Gaia within 8 arcsec. Symbol colors are otherwise the same as in Fig. 15.

Accretion in the intermediate polars may occur through streams and disks. The disks may also be large and extend up to the Roche lobe of the accreting white dwarf (Hellier et al. 1991). The freely spinning white dwarf in an IP typically leads to very rich power spectra, in particular at X-ray wavelengths, in which orbital, spin, and different types of beat periods can be traced (dependent on the orbital inclination and the accretion mode, respectively, see, e.g., Norton et al. 1996). The high degree of X-ray variability through self-eclipses and foreshortening of the accretion spots and internal absorption by accretion curtains is common to all IPs. The observed large X-ray variability within eRASS2 and between the two SRG surveys may thus argue for an IP classification. Moreover, the SAAOdiscovered period of $35 \mathrm{~min}$ and the TESS-discovered period at 25 min might support this view because if these periods are associated with the spin of the white dwarf, they are typical of IPs. In particular, the TESS-discovered periods at $25 \mathrm{~min}$ indicate an IP nature because many IPs are observed to cluster around period ratios of $P_{\text {spin }} / P_{\text {orb }}=0.1$ (Norton et al. 2004, their figure 1), as implied for SRGt $062340.2-265715$. To firmly classify the object as an IP, however, the detection of a stable spin period is required, which is lacking. The appearance and disappearance of periods at around half an hour is perhaps difficult to explain in either classification.

Optical power spectra of IPs are, however, affected by reprocessed radiation. Better insight is possible from a decent uninterrupted X-ray observation to uncover the intrinsic variability of the source on short timescales. The existing X-ray observations of SRGt 062340.2-265715 with SRG are insufficient to detect a $\sim 2000$ s period. This will require an extensive future observation (e.g., with XMM-Newton).

The X-ray spectrum of SRGt 062340.2-265715 is not too strongly absorbed and can be modeled with a single purely thermal emission component. No separate soft X-ray component was found, although the appearance of the Bowen blend appears to imply the existence of such a component. The eRASS2 bolometric X-ray flux implies an accretion luminosity of $L_{\mathrm{X}, \text { bol }}=$ $4.8 \times 10^{32} \mathrm{erg} \mathrm{s}^{-1}$, which is at the low end for an IP and at the high end for an UX UMa system. 
In addition to the color-magnitude diagram of Fig. 15, it is instructive to locate SRGt 062340.2-265715 in an X-ray to optical color-color diagram based on eROSITA and Gaia, which is shown in Fig. 16. The diagrams were constructed in the following manner. The collaboration-internal preliminary eRASS2 catalog was matched to within the 8 arcsec FHWM of the point spread functions of the telescope modules of eROSITA with Gaia DR2. For the matching objects, distances were determined following Bailer-Jones et al. (2021). We then used the final edition of the Ritter \& Kolb catalog (Ritter \& Kolb 2003) to locate the CVs. The optically bright novalike CVs of the UX UMa type are identified with a different color in the diagrams. We also used the compilation of Koji Mukai ${ }^{4}$ to highlight the IPs that were identified in eRASS. The color $B p-R p$ was taken directly from Gaia data. The X-ray to optical color was computed as $\log \left(F_{\mathrm{X}}(0.6-2.3 \mathrm{keV})+\right.$ phot_g_mean_mag $/ 2.5+4.86$, and the chosen energy band was the standard band 2 in the current version of the catalog pipeline (pipeline version 946 Brunner, $\mathrm{H}$. et al. 2021, submitted to A\&A).

Our Fig. 15 is to be compared with Fig. 2 of Abril et al. (2020), which locates the CV sub-families in the Gaia-DR2 HRD. Whether the floor of objects in Fig. 15 at $G=13$ mag indicates real associations or is due to random associations needs to be determined. Most CVs are located between the white dwarf sequence and the main sequence. Their X-ray luminosities are orders of magnitude higher than those of coronally active stars on the main sequence. The novalike CVs, in particular the magnetic IPs, overlap in the color-magnitude diagram with coronally active stars, but they can be discerned through their much higher $\mathrm{X}$-ray luminosity.

The color-color diagram of Fig. 16 has two main structures. The L-shaped sequence beginning at $(0.6,-5.5)$ with the knee at $(1,-3)$ delineates active stars, whereas the cloud around $(1,-0.5)$ is the domain of the active galactic nuclei. Most of the CVs cannot be distinguished from the numerous active galactic nuclei in this diagnostic diagram as long as no astrometric information can be used for a further distinction of galactic and extragalactic objects (see also Comparat et al. 2020, their Fig. 11).

The transient CV SRGt 062340.2-265715 is located in very sparsely populated areas in both diagrams. It optically belongs to the most luminous known X-ray detected CVs. It is bluer than most nonmagnetic UX UMa novalike CVs and bluer than all IPs, although it is not too much separated from the extreme representatives of these two classes. On the other hand, its X-ray to optical color is between that of the UX UMas and the IPs. For an IP, SRGt 062340.2-265715 is X-ray underluminous by about 1 dex; for a nonmagnetic novalike, it is also overluminous by about 1 dex.

At a Gaia magnitude of $12.5 \mathrm{mag}$, SRGt 062340.2-265715, if identified as an IP, would be the IP with the brightest apparent magnitude in eRASS2 by far. The next brightest IP is TV Col at phot_g_mean_mag $=13.9$ mag. For comparison, the first soft $\mathrm{X}$-ray discovered IP in the ROSAT all-sky survey, PQ Gem, was observed at phot_g_mean_mag $=14.1 \mathrm{mag}$.

Although only a few objects are found in the vicinity of SRGt 062340.2-265715 in the diagnostic diagrams of Figs. 15 and 16 , several candidates are located around our new discovery that will be identified in the next years with the large-scale identification programs of serendipituous eROSITA sources with the SDSS (Kollmeier et al. 2017), for instance. The journey has just begun.

\footnotetext{
${ }^{4}$ https://asd.gsfc.nasa.gov/Koji.Mukai/iphome/iphome.html
}

Acknowledgements. We thank the referee, John Thorstensen, for constructive criticism. MPM would like to thank Tom Maccarone for useful discussions. This work is based on data from eROSITA, the soft instrument aboard SRG, a joint Russian-German science mission supported by the Russian Space Agency (Roskosmos), in the interests of the Russian Academy of Sciences represented by its Space Research Institute (IKI), and the Deutsches Zentrum für Luft- und Raumfahrt (DLR). The SRG spacecraft was built by Lavochkin Association (NPOL) and its subcontractors, and is operated by NPOL with support from the Max Planck Institute for Extraterrestrial Physics (MPE). The development and construction of the eROSITA X-ray instrument was led by MPE, with contributions from the Dr. Karl Remeis Observatory Bamberg \& ECAP (FAU ErlangenNuernberg), the University of Hamburg Observatory, the Leibniz Institute for Astrophysics Potsdam (AIP), and the Institute for Astronomy and Astrophysics of the University of Tübingen, with the support of DLR and the Max Planck Society. The Argelander Institute for Astronomy of the University of Bonn and the Ludwig Maximilians Universität Munich also participated in the science preparation for eROSITA. The eROSITA data shown here were processed using the eSASS/NRTA software system developed by the German eROSITA consortium. The Mikhail Pavlinsky ART-XC telescope is the hard X-ray instrument on board the $S R G$ observatory, a flagship astrophysical project of the Russian Federal Space Program realized by the Russian Space Agency, in the interests of the Russian Academy of Sciences. ART-XC was developed by the Space Research Institute (IKI, Moscow) and the Russian Federal Nuclear Center - All-Russian Scientific Research Institute for Experimental Physics (RFNC-VNIIEF, Sarov) with the participation of the NASA's Marshall Space Flight Center (MSFC). The ART-XC team thanks the Russian Space Agency, Russian Academy of Sciences and State Corporation Rosatom for the support of the $S R G$ project and ART-XC telescope. Some of the observations presented here were obtained with SALT under the transients followup programme 2018-2-LSP-001 (PI: DB), which is supported by Poland under grant no. MNiSW DIR/WK/2016/07. DB and LT also acknowledge research support from the National Research Foundation. We acknowledge the use of TESS High Level Science Products (HLSP) produced by the Quick-Look Pipeline (QLP) at the TESS Science Office at MIT, which are publicly available from the Mikulski Archive for Space Telescopes (MAST). Funding for the TESS mission is provided by NASA's Science Mission directorate. This work has made use of data from the Asteroid Terrestrial-impact Last Alert System (ATLAS) project. The Asteroid Terrestrial-impact Last Alert System (ATLAS) project is primarily funded to search for near earth asteroids through NASA grants NN12AR55G, 80NSSC18K0284, and 80NSSC18K1575; byproducts of the NEO search include images and catalogs from the survey area. This work was partially funded by Kepler/K2 grant J1944/80NSSC19K0112 and HST GO-15889, and STFC grants ST/T000198/1 and ST/S006109/1. The ATLAS science products have been made possible through the contributions of the University of Hawaii Institute for Astronomy, the Queen's University Belfast, the Space Telescope Science Institute, the South African Astronomical Observatory, and The Millennium Institute of Astrophysics (MAS), Chile. This paper includes data collected with the TESS mission, obtained from the MAST data archive at the Space Telescope Science Institute (STScI). Funding for the TESS mission is provided by the NASA Explorer Program. STScI is operated by the Association of Universities for Research in Astronomy, Inc., under NASA contract NAS 5-26555.

\section{References}

Abril, J., Schmidtobreick, L., Ederoclite, A., \& López-Sanjuan, C. 2020, MNRAS, 492, L40

Astropy Collaboration, Price-Whelan, A. M., Sipőcz, B. M., et al. 2018, AJ, 156, 123

Bailer-Jones, C. A. L., Rybizki, J., Fouesneau, M., Demleitner, M., \& Andrae, R. 2021, AJ, 161, 147

Balman, Ş. 2020, Advances in Space Research, 66, 1097

Baluev, R. V. 2008, MNRAS, 385, 1279

Beuermann, K. \& Thomas, H. C. 1990, A\&A, 230, 326

Bowman, D. M. \& Holdsworth, D. L. 2019, A\&A, 629, A21

Brunner, H., Liu, T., Lamer, G., et al. 2021, A\&A, submitted

Buckley, D. A. H., Swart, G. P., \& Meiring, J. G. 2006, in Proc. SPIE, Vol. 6267, Society of Photo-Optical Instrumentation Engineers (SPIE) Conference Series, $62670 Z$

Childress, M., Vogt, F., Nielsen, J., \& Sharp, R. 2014, PyWiFeS: Wide Field Spectrograph data reduction pipeline

Comparat, J., Merloni, A., Dwelly, T., et al. 2020, A\&A, 636, A97

Coppejans, R., Gulbis, A. A. S., Kotze, M. M., et al. 2013, PASP, 125, 976

Cornelisse, R., Casares, J., Muñoz-Darias, T., et al. 2008, in American Institute of Physics Conference Series, Vol. 1010, A Population Explosion: The Nature \& Evolution of X-ray Binaries in Diverse Environments, ed. R. M. Bandyopadhyay, S. Wachter, D. Gelino, \& C. R. Gelino, 148-152 
Crause, L. A., Sharples, R. M., Bramall, D. G., et al. 2014, in Society of PhotoOptical Instrumentation Engineers (SPIE) Conference Series, Vol. 9147, Proc. SPIE, 91476T

Crawford, S. M., Still, M., Schellart, P., et al. 2010, in Proc. SPIE, Vol. 7737, Observatory Operations: Strategies, Processes, and Systems III, 773725

Czesla, S., Schröter, S., Schneider, C. P., et al. 2019, PyA: Python astronomyrelated packages

Dobrotka, A., Ness, J. U., Mineshige, S., \& Nucita, A. A. 2017, MNRAS, 468, 1183

Dopita, M., Rhee, J., Farage, C., et al. 2010, Ap\&SS, 327, 245

Drake, A. J., Djorgovski, S. G., Mahabal, A., et al. 2009, ApJ, 696, 870

Green, G. M., Schlafly, E., Zucker, C., Speagle, J. S., \& Finkbeiner, D. 2019, ApJ, 887, 93

Harlaftis, E. T. \& Horne, K. 1999, MNRAS, 305, 437

Heinze, A. N., Tonry, J. L., Denneau, L., et al. 2018, AJ, 156, 241

Hellier, C., Cropper, M., \& Mason, K. O. 1991, MNRAS, 248, 233

Hernandez, M. S., Zharikov, S., Neustroev, V., \& Tovmassian, G. 2017, MNRAS, 470, 1960

HI4PI Collaboration, Ben Bekhti, N., Flöer, L., et al. 2016, A\&A, 594, A116

Huang, C. X., Vanderburg, A., Pál, A., et al. 2020, Research Notes of the American Astronomical Society, 4, 204

Jenkins, J. M., Twicken, J. D., McCauliff, S., et al. 2016, in Society of PhotoOptical Instrumentation Engineers (SPIE) Conference Series, Vol. 9913, Software and Cyberinfrastructure for Astronomy IV, ed. G. Chiozzi \& J. C. Guzman, 99133E

Kniazev, A. Y., Gvaramadze, V. V., \& Berdnikov, L. N. 2016, MNRAS, 459, 3068

Kochanek, C. S., Shappee, B. J., Stanek, K. Z., et al. 2017, PASP, 129, 104502

Kollmeier, J. A., Zasowski, G., Rix, H.-W., et al. 2017, arXiv e-prints, arXiv: 1711.03234

Lightkurve Collaboration, Cardoso, J. V. d. M., Hedges, C., et al. 2018 , Lightkurve: Kepler and TESS time series analysis in Python, Astrophysics Source Code Library

Lomb, N. R. 1976, Ap\&SS, 39, 447

Masci, F. J., Laher, R. R., Rusholme, B., et al. 2019, PASP, 131, 018003

Mauche, C. W. \& Mukai, K. 2002, ApJ, 566, L33

Mukai, K. 2017, PASP, 129, 062001

Norton, A. J., Beardmore, A. P., \& Taylor, P. 1996, MNRAS, 280, 937

Norton, A. J., Wynn, G. A., \& Somerscales, R. V. 2004, ApJ, 614, 349

Pavlinsky, M., Tkachenko, A., Levin, V., et al. 2021, arXiv e-prints, arXiv:2103.12479

Pichardo-Marcano, M. 2020, The Astronomer's Telegram, 14222, 1

Predehl, P., Andritschke, R., Arefiev, V., et al. 2021, A\&A, 647, A1

Ricker, G. R., Winn, J. N., Vanderspek, R., et al. 2014, in Society of PhotoOptical Instrumentation Engineers (SPIE) Conference Series, Vol. 9143, Space Telescopes and Instrumentation 2014: Optical, Infrared, and Millimeter Wave, ed. J. Oschmann, Jacobus M., M. Clampin, G. G. Fazio, \& H. A. MacEwen, 914320

Ritter, H. \& Kolb, U. 2003, A\&A, 404, 301

Scargle, J. D. 1982, ApJ, 263, 835

Schachter, J., Filippenko, A. V., Kahn, S. M., \& Paerels, F. B. S. 1991, ApJ, 373, 633

Schwope, A. 2012, Mem. Soc. Astron. Italiana, 83, 844

Schwope, A., Semena, A., Maitra, C., et al. 2020, The Astronomer's Telegram, 14219,1

Shappee, B. J., Prieto, J. L., Grupe, D., et al. 2014, ApJ, 788, 48

Smith, J. C., Stumpe, M. C., Van Cleve, J. E., et al. 2012, PASP, 124, 1000

Stellingwerf, R. F. 1978, ApJ, 224, 953

Sunyaev, R., Arefiev, V., Babyshkin, V., et al. 2021, arXiv e-prints, arXiv:2104.13267

Tonry, J. L., Denneau, L., Heinze, A. N., et al. 2018, PASP, 130, 064505

Zemko, P., Orio, M., Mukai, K., \& Shugarov, S. 2014, MNRAS, 445, 869 


\title{
Identification of SRGt 062340.2-265715 as a bright, strongly variable, nova-like cataclysmic variable
}

\author{
Axel Schwope $^{1}$, David A.H. Buckley ${ }^{2,3}$, Adela Kawka ${ }^{4}$, Ole König ${ }^{5}$, Alexander Lutovinov ${ }^{6}$, Chandreyee Maitra ${ }^{7}$, Ilya \\ Mereminskiy $^{6}$, James Miller-Jones ${ }^{4}$, Manuel Pichardo Marcano ${ }^{8}$, Arne Rau ${ }^{7}$, Andrei Semena ${ }^{6}$, Lee J. Townsend ${ }^{2}$, and \\ Jörn Wilms ${ }^{5}$ \\ ${ }^{1}$ Leibniz-Institut für Astrophysik Potsdam (AIP), An der Sternwarte 16, 14482 Potsdam, Germany \\ e-mail: aschwope@aip.de \\ 2 South African Astronomical Observatory, P.O. Box 9, Observatory, 7935, Cape Town, South Africa \\ 3 Department of Astronomy, University of Cape Town, Private Bag X3, Rondebosch 7701, South Africa \\ 4 International Centre for Radio Astronomy Research, Curtin University, GPO Box U1987, Perth, WA 6845, Australia \\ 5 Dr. Karl Remeis-Sternwarte \& Erlangen Centre for Astroparticle Physics, Friedrich-Alexander-Universität Erlangen-Nürnberg, \\ Sternwartstr. 7, 96049 Bamberg, Germany \\ 6 Space Research Institute (IKI) of Russian Academy of Sciences, Profsoyuznaya ul 84/32, 117997 Moscow, Russian Federation \\ 7 Max-Planck-Institut für extraterrestrische Physik, Gießenbachstraße, 85748 Garching, Germany \\ 8 Department of Physics and Astronomy, Texas Tech University, USA
}

June 29, 2021

\begin{abstract}
We report the identification and follow-up of the transient SRGt 062340.2-265715 detected with both instruments onboard the Spektrum-Roentgen-Gamma mission. Optical spectroscopy of the $G=12.5$ counterpart firmly classify the object as nova-like cataclysmic variable $(\mathrm{CV})$ at a distance of $495 \mathrm{pc}$. A highly significant TESS period of 3.941 hours, tentatively identified with the orbital period of the binary, could not be found when the object was re-observed with TESS two years later. The newer high-cadence TESS data revealed quasi-periodic oscillations around $25 \mathrm{~min}$, while ground-based photometry indicated periodic variability at $32 \mathrm{~min}$. Located in very sparsely populated regions of color-magnitude diagrams involving X-ray and optical magnitudes and colors, the new object could be an X-ray underluminous magnetic CV, an intermediate polar, or an overluminous non-magnetic CV. The lack of uniquely identified spin and orbital periods prevents a final classification. The site of X-ray production in the system, $L_{\mathrm{X}, \mathrm{bol}}=4.8 \times 10^{32} \mathrm{erg}$ $\mathrm{s}^{-1}$, remains yet to be understood given its high variability on long and short time-scales.
\end{abstract}

Key words. stars: cataclysmic variables - X-rays: stars - stars: individual: SRGt 062340.2-265715

\section{Introduction}

In the course of the second all-sky survey, on 2020 October 12 both instruments on board the Spektrum-Roentgen-Gamma mission ( $S R G$, Sunyaev et al. 2021) detected a bright transient at galactic coordinates $l^{I I}=234.7, b^{I I}=-17.6$. The comparison of data obtained during the first and the second all-sky surveys with both instruments onboard the mission, the Mikhail Pavlinsky ART-XC (Pavlinsky et al. 2021) and eROSITA (Predehl et al. 2021), revealed an increase of the X-ray flux of about a factor 2 , which triggered some fast-turnaround follow-up observations.

An initial announcement of the source was published by Schwope et al. (2020) and some analysis of the TESS data by Pichardo-Marcano (2020). In this paper we describe the initial results in more detail together with follow-up spectroscopy and photometry from the ground and space. The transient was found to be coincident with catalogued ROSAT, Swift/XRT, and Gaia sources (1RXS J062339.8-265744, 2SXPS J062339.9-265751), and the optical transient ZTF19aaabzuh, respectively. It was mentioned earlier as a cataclysmic variable by Denisenko on his webpage ${ }^{1}$ but no further supporting information was available.

\footnotetext{
${ }^{1}$ http://scan.sai.msu.ru/ denis/VarDDE.html
}

The X-ray and optical observations uniquely identify the transient with a nova-like cataclysmic variable $(\mathrm{CV})$. CVs are close binaries with white dwarfs accreting from a Roche-lobe filling star. In gross terms, they may physically be divided into magnetic and non-magnetic objects (disk accretion vs. quasiradial accretion), phenomenologically they are often sorted into dwarf novae (DN, non-magnetic, disk accreting CVs which may become unstable and cause the DN outburst) and so-called novalikes. Historically both magnetic and non-magnetic CVs were sorted into this last bin. The non-magnetic, disk-accreting novalikes (often referred to as UX UMas although the VY Scl, Z Cam, SW Sex objects and possibly other subtypes may also fall in this class) are high-accretion rate objects whose disks are permanently found in the hot state so that no dwarf nova outbursts are launched. CVs of all subtypes were expected to be found in large number through systematic spectroscopic followup of point-like sources serendipituously discovered in the ongoing all-sky surveys with eROSITA (Schwope 2012). The case considered here is unusual through its very high optical brightness and its high and strongly variable $\mathrm{X}$-ray brightness also in the hard $S R G$ band which allowed its discovery with both instruments on the spacecraft. 


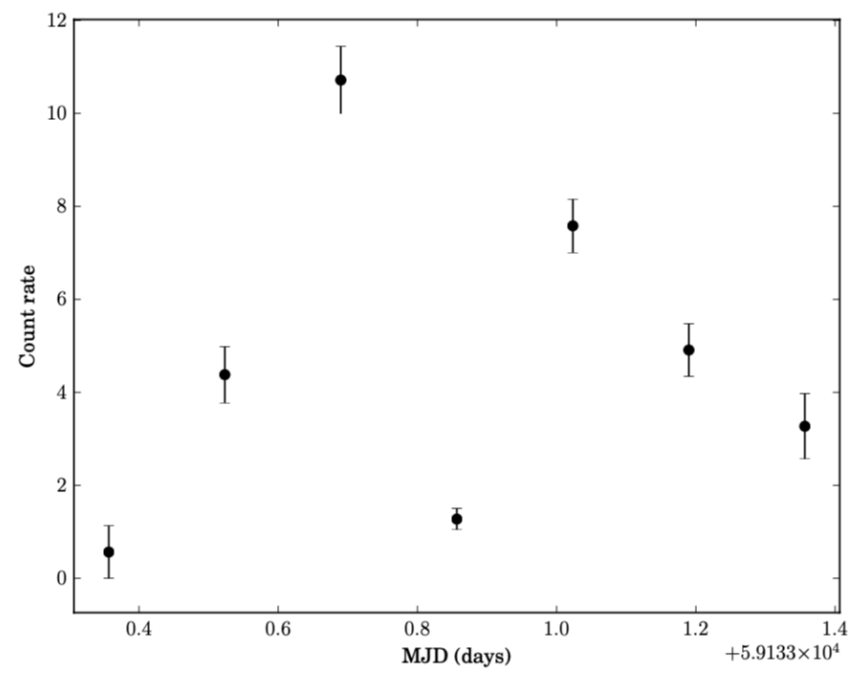

Fig. 1. X-ray light curve of SRGt 062340.2-265715 obtained for eRASS2 $(0.2-10 \mathrm{keV})$. Each data point represents the mean rate of one individual scan over the source position.

\section{Observations and analysis}

\section{1. $S R G$}

The transient of 2020 October 12 was observed at the $e$ ROSITA position $\alpha_{\mathrm{J} 2000.0}=06^{\mathrm{h}} 23^{\mathrm{m}} 40 \mathrm{~s} 2(95.91754), \delta_{\mathrm{J} 2000.0}=$ $-26^{\circ} 57^{\prime} 15^{\prime \prime}(-26.96425)$ with a positional uncertainty of 1 '.6 (0".6 statistical, 1".5 systematic). The Mikhail Pavlinsky ART$\mathrm{XC}$ telescope detected the source at the position of $\alpha_{\mathrm{J} 2000.0}=$ $06^{\mathrm{h}} 23^{\mathrm{m}} 39.7, \delta_{\mathrm{J} 2000.0}=-26^{\circ} 57^{\prime} 53^{\prime \prime}$ with an uncertainty of $15^{\prime \prime}$ (90\%).

During the second $S R G$ survey the object had a total exposure time of $85 \mathrm{~s}$ (an effective exposure time of $25 \mathrm{~s}$ ) with ART-XC and was discovered at a mean flux of $1.3_{-0.3}^{+0.4} \times$ $10^{-11} \mathrm{erg} \mathrm{cm}^{-2} \mathrm{~s}^{-1}$ in the $4-12 \mathrm{keV}$ energy band. It was below the detection threshold during the first survey, when it had an effective exposure time of $21 \mathrm{~s}$, corresponding to an upper limit for the 4-12 keV flux of $F<10^{-11} \mathrm{erg} \mathrm{cm}^{-2} \mathrm{~s}^{-1}$. With eROSITA, the source was detected in both surveys, in eRASS1 (2020 April 8 ), where it received a total exposure time of $228 \mathrm{~s}$, with a mean count rate of $1.38 \pm 0.11 \mathrm{~s}^{-1}$ and in eRASS2 (2020 October 12) with a mean rate of $5.84 \pm 0.24 \mathrm{~s}^{-1}$ for a total exposure of $208 \mathrm{~s}$.

More than 1200 photons were collected during eRASS2 which allowed to generate an eRASS2 light curve which is displayed in Fig. 1. Circular regions with radii of $75^{\prime \prime}$ and $100^{\prime \prime}$ were used for the source and background extraction, respectively. SRGt 062340.2-265715 was observed during seven erodays $^{2}$ and showed variability between $10.7 \pm 0.7 \mathrm{~s}^{-1}$ and $1.3 \pm 0.2 \mathrm{~s}^{-1}$. Most of the photons registered with ART-XC were registered during the fourth and the fifth eroday (see Fig. 1).

The combined spectrum from both instruments onboard SRG is displayed in Fig. 2. It was fit using XSPEC version 12.11 with a one-component emission model, a thermal plasma absorbed by cold interstellar matter. A scaling factor between the two spectral groups was also fit that accounts (a) for calibration uncertainties between the two involved instruments and (b) for the fact that the data were not taken strictly simultaneously which is impor-

\footnotetext{
${ }^{2}$ An eroday lasts 4 hours and corresponds to the revolution of the spacecraft around the scan axis. The exposure for a given sky position is different for each eroday and may last up to $40 \mathrm{sec}$ dependent on the off-axis angle
}

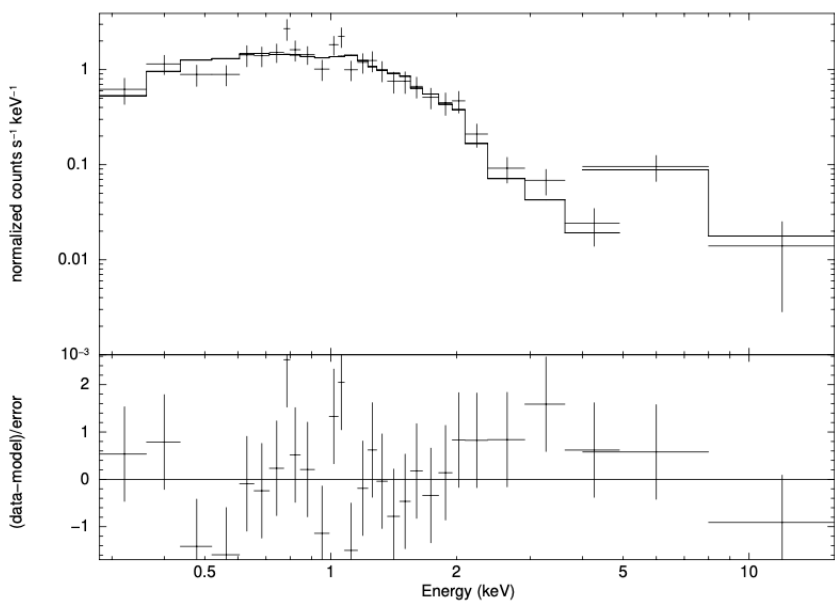

Fig. 2. Mean combined spectrum of ART X-C and eROSITA of SRGt 062340.2-265715 obtained during the second survey with SRG with the best-fitting thermal model spectrum. The lower panel shows the residuals in units of the error per spectral bin.

tant given the high variability of the source. The C-statistic was used for fitting, the best-fit value found was 180 for 230 bins. This provides a temperature $k T=5.7_{-1.6}^{+2.6} \mathrm{keV}$ (errors given for a $90 \%$ confidence interval $)$ and fluxes of $F_{\text {eRO }}(0.5-2.0 \mathrm{keV})=$ $4.3_{-0.4}^{+0.3} \times 10^{-12} \mathrm{erg} \mathrm{cm}^{-2} \mathrm{~s}^{-1}, F_{\mathrm{eRO}}(0.5-10 \mathrm{keV})=1.2_{-0.4}^{+0.5} \times$ $10^{-11} \mathrm{erg} \mathrm{cm}^{-2} \mathrm{~s}^{-1}$ and $F_{\mathrm{ART}}(4-12 \mathrm{keV})=2.0_{-0.3}^{+0.14} \times 10^{-11}$ erg $\mathrm{cm}^{-2} \mathrm{~s}^{-1}$, respectively. The column density was determined using the TBabs command (abundance set to wilms) but was not found to be well constrained, therefore it was fixed at the Galactic column density in this direction of $\mathrm{N}_{\mathrm{H}}=4.08 \times 10^{20} \mathrm{~cm}^{-2}$ (HI4PI Collaboration et al. 2016). Recent 3D dust maps (e.g., Green et al. 2019) do not provide better constraints due to rather large errors, $E(g-r)=0.03 \pm 0.02$. The implied column density is compatible with that inferred from X-rays. The same model applied to the eRASS1 data with fixed amount of interstellar absorption gave a lower temperature $k T=2.0_{-0.6}^{+3.4} \mathrm{keV}$ at a flux of $F_{\mathrm{X}}(0.5-2.0 \mathrm{keV})=(8.4 \pm 1.6) \times 10^{-13} \mathrm{erg} \mathrm{cm}^{-2} \mathrm{~s}^{-1}$. The bolometric flux of the best-fitting model during the high state in October 2020 was $F_{\mathrm{X}}=1.6 \times 10^{-11} \mathrm{erg} \mathrm{cm}^{-2} \mathrm{~s}^{-1}$ (eROSITA value).

\subsection{Gaia}

Gaia EDR3 lists an object (source_ID 2899766827964264192) at a distance of 2.4 from the eRASS2 catalog position of the transient at position RA $=95.91672681385$, DEC $=$ -26.96405593108 . A finding chart indicating the eRASS2 Xray position and all Gaia sources in its vicinity is reproduced in Fig. 3. It has a parallax of $\pi=1.9749 \pm 0.0192$ mas and photometric properties $G=12.436 \pm 0.005 \mathrm{mag}, B p-R p=0.023$. Following Bailer-Jones et al. (2021) the median geometric distance to SRGt $062340.2-265715$ is $495.5 \pm 4$ pc which gives an absolute magnitude $M(G)=3.96$.

\subsection{Archival $X$-ray observations}

The position of SRGt $062340.2-265715$ was covered previously by past (ROSAT) and current (XMM-Newton, Swift/XRT) X-ray missions. The corresponding information is available via the ESA-supported high-energy light curve generator HILIGT (up- 
Table 1. Archival X-ray observations of SRGt 062340.2-265715 as derived from the ESA upper limit server HILIGT

\begin{tabular}{lcrlll}
\hline \hline Mission & Date & Exp time & Flux $^{a}$ & Flux $^{a}$ & Flux $^{a}$ \\
& & $(\mathrm{~s})$ & $0.2-2 \mathrm{keV}$ & $2-12 \mathrm{keV}$ & $0.2-12 \mathrm{keV}$ \\
\hline ROSAT-Survey & $1990-09-1900: 47: 17$ & 661.5 & $2.1 \pm 0.2$ & & \\
XMM-Newton slew & $2003-04-01$ & 5.6 & $2.3 \pm 0.8$ & $<28.2$ & $6.4 \pm 2.0$ \\
XMM-Newton slew & $2006-10-02$ & 9.9 & $5.0 \pm 0.8$ & $11.6 \pm 3.3$ & $12.4 \pm 1.8$ \\
Swift-XRT & $2008-07-17$ & 5558.3 & $1.7 \pm 0.1$ & $2.3 \pm 0.2$ & $3.8 \pm 0.2$ \\
\hline
\end{tabular}

${ }^{a}$ Absorbed flux in units of $10^{-12} \mathrm{erg} \mathrm{cm}^{-2} \mathrm{~s}^{-1}$. Count rate to flux conversion performed with the a power law spectral model of slope 2 and absorption $N_{\mathrm{H}}=3 \times 10^{20} \mathrm{~cm}^{-2}$.

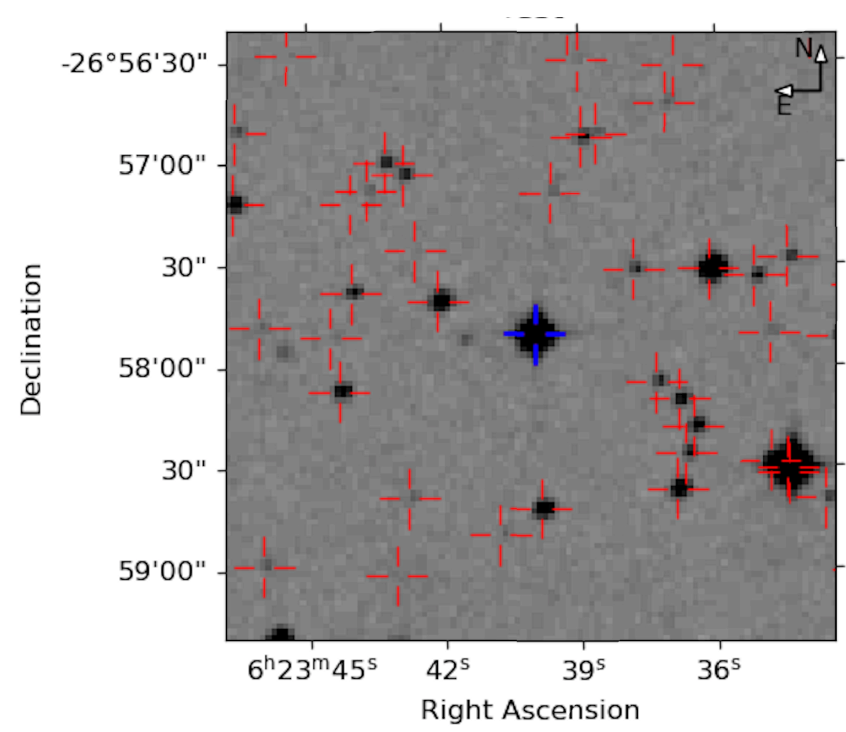

Fig. 3. Finding chart of the new transient $(3 \times 3$ arcmin). The background image is from the DSS; the eROSITA position (eRASS2) is marked with a blue cross. All further Gaia objects in the field of view are marked with red crosses.

per limit server) ${ }^{3}$ and listed in Tab. 1. Although HILIGT does not offer a thermal spectral model to convert count rates to fluxes the given values are at least indicative. The flux in the soft band, $0.2-2.0 \mathrm{keV}$, common to all the missions, was found to be variable by at least a factor 2 .

\subsection{Initial follow-up spectroscopy with WiFeS and SALT}

The likely Gaia counterpart was selected for follow-up spectroscopy with the Australian National University $2.3 \mathrm{~m}$ telescope equipped with the Wide Field Spectrograph (WiFeS; Dopita et al. 2010) and with the $10 \mathrm{~m}$-class Southern African Large Telescope (SALT; Buckley et al. 2006)) equipped with the High Resolution Spectrograph (HRS; Crause et al. 2014). WiFeS is a double-beam, image-slicing, integral-field spectrograph. Observations were performed on 2020 October 28.164 with the R3000 grating and an exposure of $300 \mathrm{~s}$. As the red arm of the instrument was not operating only the blue wavelength range between 3500 and $5500 \AA$ is available. The WiFeS spectrum was reduced using the PyWiFeS reduction pipeline (Childress et al. 2014) which produced three-dimensional data. We then extracted background subtracted spectra from the slitlets that provided significant flux.

\footnotetext{
${ }^{3}$ http://xmmuls.esac.esa.int/hiligt/
}
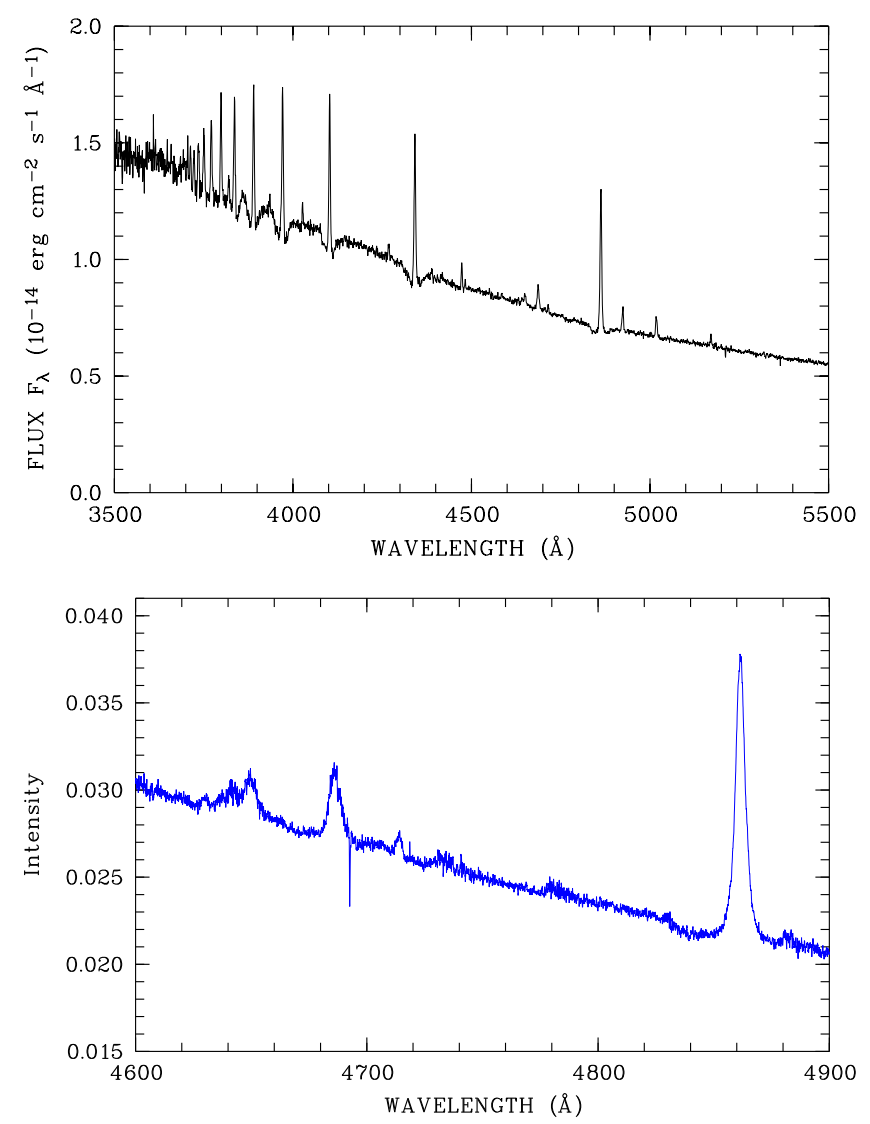

Fig. 4. ANU/WiFeS low-resolution and SALT/HRS high-resolution identification spectra of SRGt 062340.2-265715. The SALT spectrum has just arbitrary uncalibrated intensity units. Note the presence of high excitation N III $4640 \AA$ Bowen fluorescence and He II $4540 \AA$ and $4686 \AA$ lines.

HRS is a dual-beam, fibre-fed èchelle spectrograph, covering 3800-8900 A. A 1200 s HRS observation was taken on 2020 October 31.9672 in Low Resolution (LR; R 15,000) mode. Initial reduction of the HRS spectrum was achieved using the PySALT package (Crawford et al. 2010), which includes overscan correction, bias subtraction and gain correction. The spectrum extraction was carried out using the HRS pipeline, based on MIDAS routines described in Kniazev et al. (2016). The ANU/WiFeS and SALT/HRS spectra are displayed in Fig. 4.

The object has a very blue continuum that rises up to the short wavelength cutoff at $3500 \AA$. The WiFeS spectrum reveals broad Balmer absorption lines whose centers are filled with intense emission lines. Apart from $\mathrm{H}$-Balmer emission lines the object displays He-emission lines, both neutral and ionized. 
Through those early spectra the object is robustly identified as a cataclysmic variable. None of the spectra shows any sign of the donor star.

The typical FWHM of the absorption lines corresponds to $3000 \mathrm{~km} \mathrm{~s}^{-1}$, while those of the Balmer emission lines correspond to $300-380 \mathrm{~km} \mathrm{~s}^{-1}$. The ionized helium line at $4686 \AA$ is detected but appears to be rather weak. It has an equivalent width of $\sim-0.9 \AA$, a FWHM of $\sim 6 \AA\left(\sim 380 \mathrm{~km} \mathrm{~s}^{-1}\right)$ and the flux ratio to the Balmer emission line $\mathrm{H} \beta$ is $F\left(\mathrm{He}_{\mathrm{II}}\right) / F(H \beta) \simeq 0.26$, while $\mathrm{H} \beta$ has an equivalent width of $\sim-4.2 \AA$ and FWHM of $\sim 5.5 \AA\left(\simeq 340 \mathrm{~km} \mathrm{~s}^{-1}\right)$. The HRS spectrum of the region around He II 4686 also uncovers the Bowen blend of $\mathrm{N}_{\text {III }}$ lines, a feature often encountered in bright low-mass X-ray binaries (LMXBs, e.g. Cornelisse et al. 2008), but also in both kinds of magnetic CVs, the polars and the intermediate polars (e.g., Schachter et al. 1991; Harlaftis \& Horne 1999) indicating a strong UV/EUV source.

The $\mathrm{H} \beta$ emission line has a centroid of $4861.54 \AA$ in the SALT spectrum whereas it was found at $4862.59 \AA$ in the WiFeS spectrum while being similarly broad ( $5.2 \AA$ vs. $5.5 \AA$ ). The difference in wavelength corresponds to a velocity difference of $65 \mathrm{~km} \mathrm{~s}^{-1}$ illustrating the feasibility of a radial velocity study.

\subsection{TESS}

We analyze data from the Transiting Exoplanet Survey Satellite (TESS, Ricker et al. 2014). TESS, using four CCD cameras, obtains continuous optical images of a rectangular field of $24^{\circ} \times 90^{\circ}$ for 27.4 days at a short cadence ( 30 minutes to 20 seconds).

The source was observed in sector 6 , which was observed at 30 minutes cadence between MJD 58467.79 to MJD 58489.54 (2018-DEC-15 to 2019-JAN-06). The source was also observed in sector 33 at a faster 2 minute cadence between MJD 59201.24 (2020-DEC-18) and MJD 59227.07 (2021-JAN-13).

We began the analysis with the data obtained in sector 6 (30 min cadence) and used the Python package Lightkurve (v1) (Lightkurve Collaboration et al. 2018) to create the lightcurves from the full-frame images (FFI). From the FFI to extract our light curves, we select pixels around the coordinates of the target, and background, 'empty', pixels with no known Gaia sources as our background and with a median flux that is less than a given threshold. We also used the TESS light curve from the MIT Quick-Look Pipeline (QLP, Huang et al. 2020) and compared them to our light curves from the full-frame images using the median background removal method. The results from the two programs agreed well. The final extracted light curve (30 min cadence), normalized to the median value of 513.1 electrons $\mathrm{s}^{-1}$, is displayed in Fig. 5. The light curve is flat to first order but displays an apparent flickering which gives the impression of a possible periodic behaviour. We thus performed a periodogram analysis to search for any underlying periodicity.

We performed period searches using the Lomb-Scargle (LS, Lomb 1976; Scargle 1982) and the phase dispersion minimization technique (PDM, Stellingwerf 1978) as implemented in the Astropy and the PyAstronomy packages (Astropy Collaboration et al. 2018; Czesla et al. 2019). The LS-periodogram is then normalized by the residuals of the data around a constant reference model. The periodogram (Fig. 6) clearly shows an isolated peak at a period of $3.941 \pm 0.010 \mathrm{~h}$, the error being estimated from the width of the peak $(\sigma \sim 0.01 \mathrm{~h})$. We also calculate a false alarm probability of $3.9 \times 10^{-158}$ using the method described in Baluev (2008).

Article number, page 4 of 9

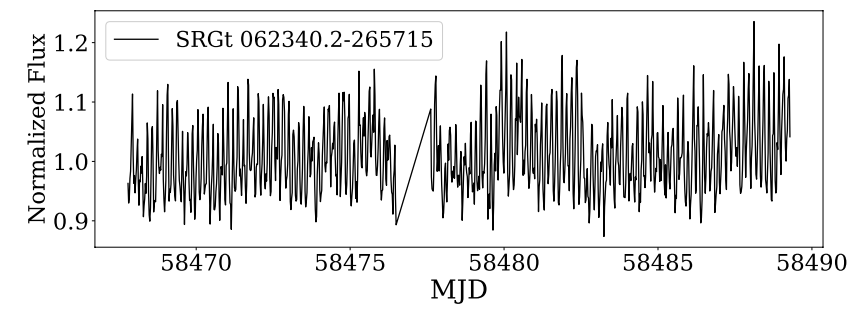

Fig. 5. TESS lightcurve for the SRGt 062340.2-265715. The normalized light curve was obtained by dividing the background corrected source flux by its median value of 513.1 electrons $\mathrm{s}^{-1}$.

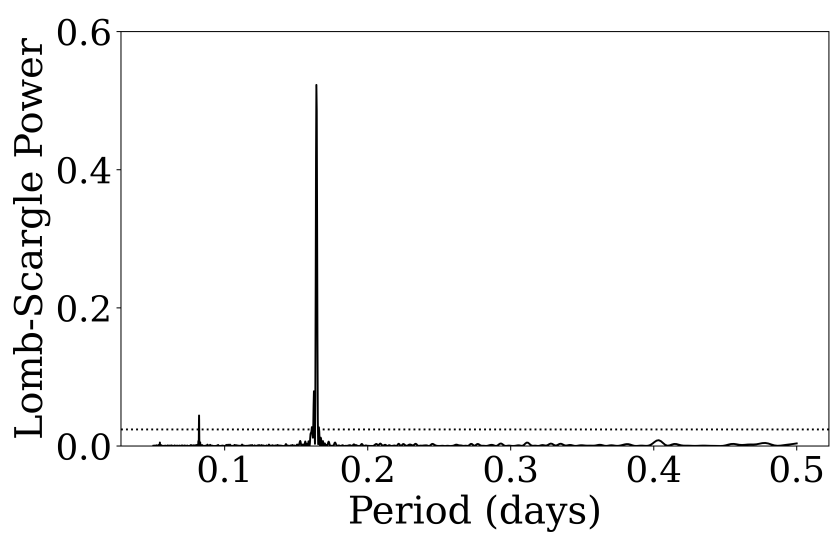

Fig. 6. Lomb-Scargle periodogram for SRGt 062340.2-265715, showing a clear isolated peak around 3.941 hours. The dotted line shows the periodogram level corresponding to a maximum peak false alarm probability of $1 \%$, using the bootstrap method that simulates data at the same observation times to approximate the true distribution of peak maxima for the case with no periodic signal present. The bootstrap method is performed with periods between 0.1 and 0.5 days, and after normalizing by the residuals of the data around a constant reference model.

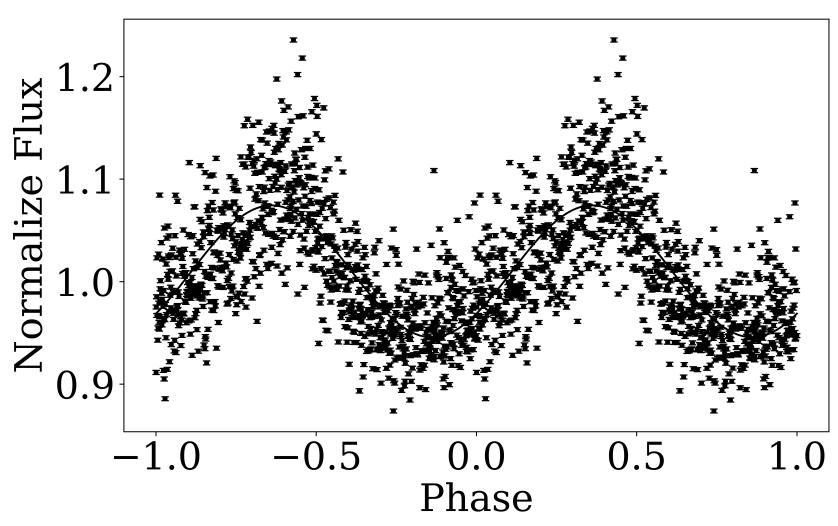

Fig. 7. TESS lightcurve for SRGt 062340.2-265715, folded on the 3.941 hours period. Data are shown with their error bars. The solid lines represents the best-fit sinusoid. Phase zero was chosen arbitrarily.

In the PDM method, the lightcurve is divided into different phase bins and the cost function, $\Theta=s^{2} / \sigma^{2}$, is minimized to choose the best period. Here $s$ is the phase bin variance and $\sigma$ is the total data variance. We divide the data into 10 equidistant bins to calculate $\Theta$ and find also a period of 3.941 hours, fully consistent with the results obtained with the Lomb-Scargle periodogram. 


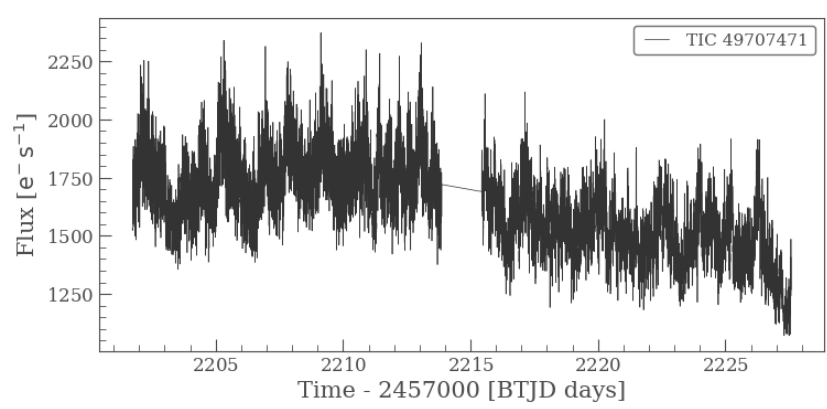

Fig. 8. TESS lightcurve for SRGt 062340.2-265715 obtained in sector 33 with 2 min cadence.

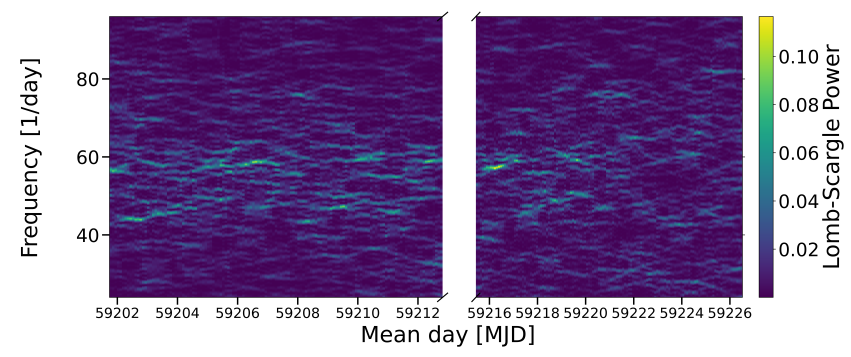

Fig. 9. Moving window periodogram of the TESS two-minute cadence data for a sliding window of 1 day length and time steps of 2 hours. The $\mathrm{x}$-axis is the middle of the 1-day window and the color corresponds to the Lomb-Scargle power. The broken axis corresponds to the dates around the middle of the light curve that has some missing data.

The main result of the analysis of the 30 min cadence data, the presence of a periodicity at about 3.9 hours, was reported very briefly already in ATEL 14222 by one of us (PichardoMarcano 2020). The more thorough analysis presented here confirms and refines the initial result. The lightcurve folded at the period of 3.941 hours is shown in Fig. 7. It is slightly skewed with a more gradual increase and a steeper decrease. There is significant scatter at any given phase which hints to further variability on shorter time scales, which could not be resolved with the low cadence TESS data of sector 6 .

For the data obtained with two-minute cadence we use the Pre-search Data Conditioning Simple Aperture Photometry flux (PDCSAP), in which long-term trends have been removed using so-called Co-trending Basis Vectors (CBVs, Smith et al. 2012), produced by the TESS Science Processing Operations Center (SPOC; Jenkins et al. 2016). The light curve of data from sector 33 is shown in original time sequence in Fig. 8. At this occasion the source appeared fundamentally different compared to the data obtained in sector 6 . At the beginning of the observations the source was about 1.4 mag brighter than two years before, at the end of this one month interval only by about 0.9 mag. A Lomb-Scargle period search for the whole dataset from sector 33 no longer showed the $3.9 \mathrm{~h}$ modulation. We then selected shorter time intervals of one or two days length at the beginning, centre, and end of the data train and searched for periodic behavior between $10 \mathrm{~min}$ and 5 hours. This revealed some power at $25 \mathrm{~min}$. To study the stability of such a period we calculated a dynamic periodogram using a time slice of one day length that was moved forward with a stepsize of 2 hours. For each slice a LS-periodogram was computed and the results arranged as a two-dimensional periodogram (LS-power as a function of time) which is shown in Fig. 9.

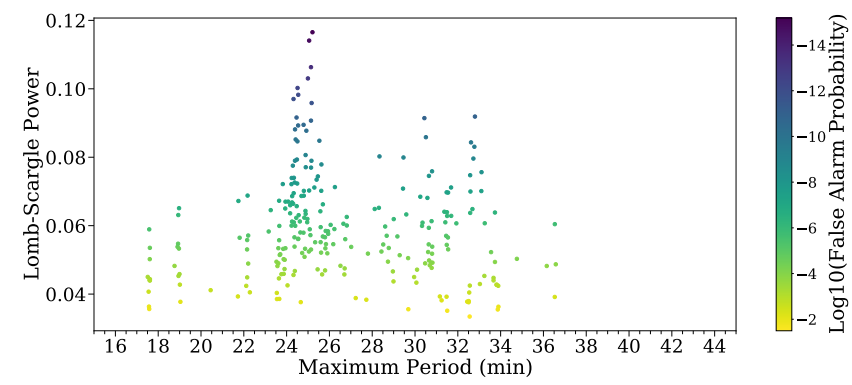

Fig. 10. For each 1 day window the maximum amplitude of the periodogram and its corresponding period is plotted. The color corresponds to the $\log _{10}$ of the FAP.

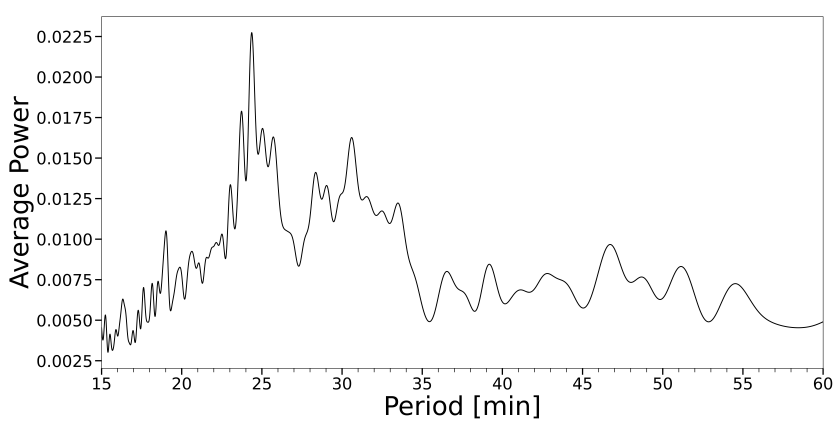

Fig. 11. Mean periodogram of sector 33 calculated by averaging all 1 day periodograms of Fig. 9.

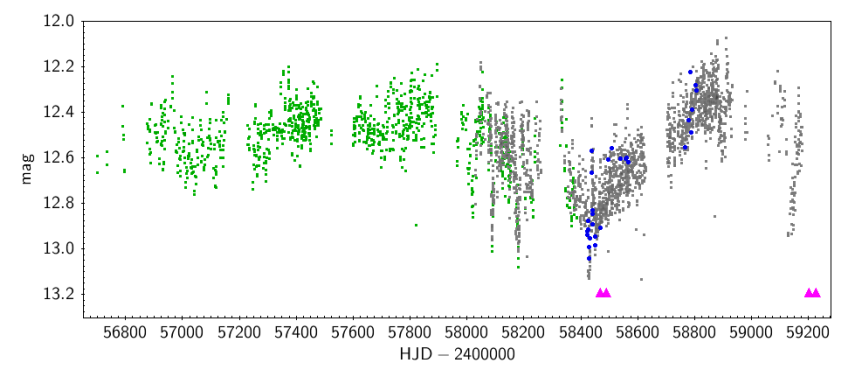

Fig. 12. ASASS-SN and ZTF photometric data for SRGt 062340.2-265715 (green symbols: ASAS-SN $V$, grey: ASASSN $g$, blue: ZTF $g$ ). The magenta triangles mark TESS observations with low and high cadence (first and second pair identify TESS sectors 6 and 33, respectively).

Complementary views on the complex timing behavior of the source are given in Figs. 10 and 11 which show the false alarm probabilities of the highest peak in each of the 1-day periodograms and the mean periodogram of all the individual 1day periodograms. We found significant power centred around $25 \mathrm{~min}$ (frequency $57.6 \mathrm{~d}^{-1}$ ), but measured significant frequencies jumping between 45 and 65 cycles per day on timescales of about one day. No significant period was found during the last quarter of the data set. The mean periodogram has maximum power at $24.37 \mathrm{~min}$, the most significant period in a 1-day time slice is observed at $25.201 \mathrm{~min}$ with a false alarm probability of $6.58 \times 10^{-16}$. 


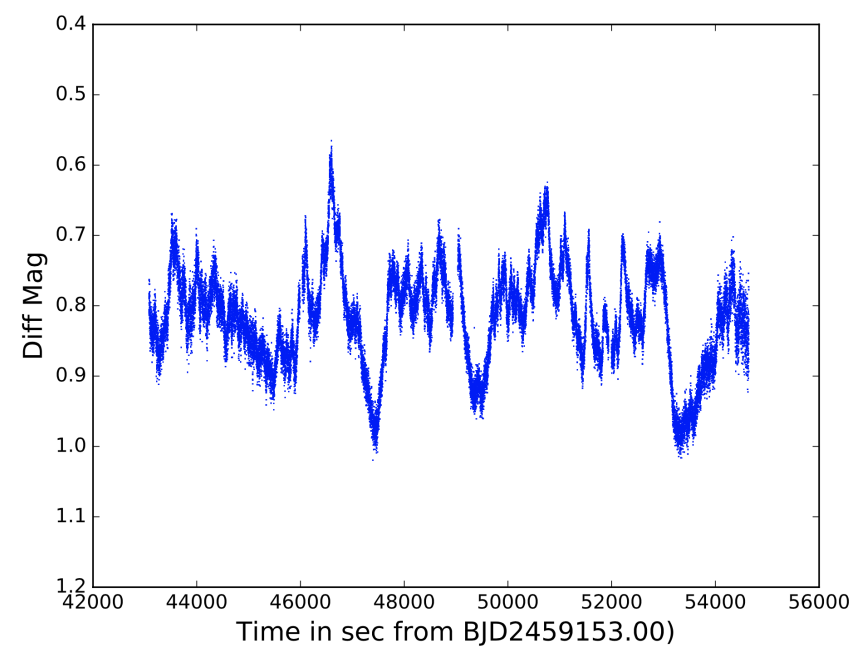

Fig. 13. Differential white-light optical light curve, spanning $3.2 \mathrm{~h}$, of SRGt $062340.2-265715$ obtained with $S A A O / S H O C$ at 0.3 s cadence. Time is seconds since 00:00 UT on 1 Nov 2020.

\subsection{Archival photometry from the CRTS, ZTF, ASAS-SN, and ATLAS}

Archival photometric information of the new transient is available from the Catalina Real-Time Transient survey (CRTS, Drake et al. 2009, 196 epochs), the Zwicky Transient Facility (ZTF, Masci et al. 2019, 55 epochs), the All-Sky Automated Survey for Supernovae (ASAS-SN, Shappee et al. 2014; Kochanek et al. 2017, 3350 epochs), and the Asteroid Terrestrial-impact Last Alert System (ATLAS, Tonry et al. 2018; Heinze et al. 2018, 34 epochs). The observations of those surveys cover the time intervals 2005-AUG-28 to 2010-APR-19, 2018-NOV-1 to 2019-NOV-18, 2014-FEB-11 to 2020-NOV-24, 2021-FEB-16 to 2021-MAR-16, respectively, and part of those are shown in Fig. 12 (omitting the CRTS and ATLAS data). ZTF data were taken in the $g$ and $r$ filters with only $g$-band data shown, and ASAS-SN data through $g$, and $V$ filters. All those data show extended phases (years) with little variability around a mean magnitude of about 12.5, with superposed scatter with amplitude of 0.1 mag followed by dimmed phases with a larger short-term variability amplitude. Such excursions towards lower brightness were observed two times, one at the end of the CRTS data train at MJD 56350, the other covered by both ZTF (partially) and ASAS-SN at MJD 58400. The minimum brightness of such fainter states is at around $13 \mathrm{mag}$. TESS observations in sector 6 revealing the 3.9 hour periodicity were obtained at the pronounced minimum. No automated survey data (like ASAS-SN, ATLAS) are available at time of writing when TESS observed the source again in sector 33 .

We searched for periodic behavior of the source in the ASAS-SN data. We selected $g$-band data obtained between HJD 2458455 and 2458866. During this 400 day interval with 1072 data points the transient recovered from its faint state at $g=12.95$ to a bright state at $g=12.3$ with a constant gradient. The brightness displayed a scatter with a mean amplitude of 0.085 mag around the trend, while the mean magnitude error was only $0.01 \mathrm{mag}$. We used the LS period search method as implemented in ESO-MIDAS and found a period $3.9142 \pm 0.0011$ hours, consistent with the value found in the TESS data at $2.5 \sigma$.

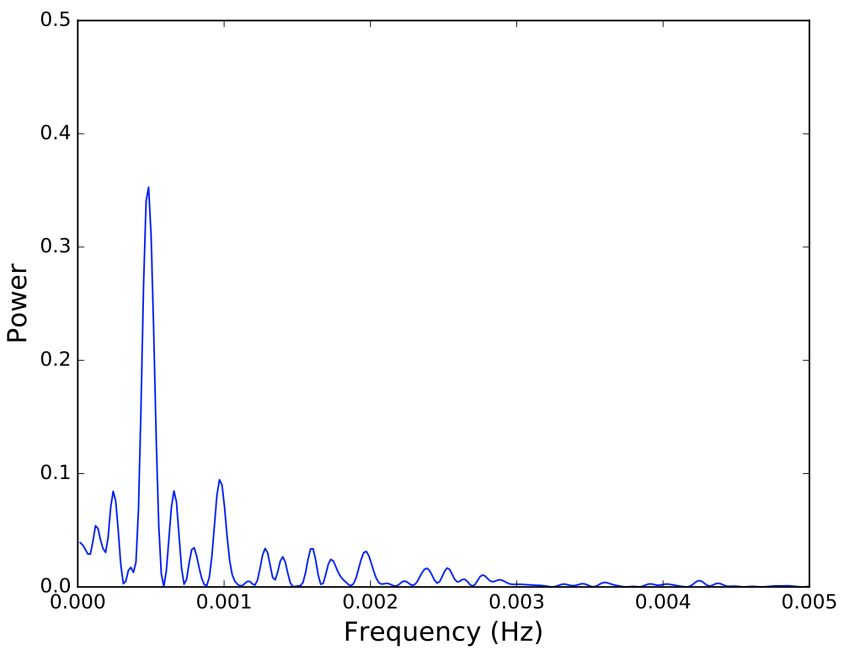

Fig. 14. Lomb-Scargle power spectrum of the light curve of SRGt 062340.2-265715 (shown in Fig 13) indicating a possible $\sim 2100$ s periodicity.

\subsection{SAAO High-speed photometry}

Following its spectral identification (see subsection 2.4), we performed $3.2 \mathrm{~h}$ of high-speed photometry with the South African Astronomical Observatory 1.0-m telescope on 2020 Nov 1, beginning at 23:57:30 UTC. The Sutherland High speed Optical Camera (SHOC; Coppejans et al. 2013) CCD camera, which uses an Andor iXon888 frame transfer EM-CCD frame camera $(1024 \times 1024$ pixels $)$ was used. The observations were done without a filter (i.e., 'white light') with an exposure time of $0.3 \mathrm{~s}$.

The CCD images were reduced using the TEAPhot photometry reduction package (Bowman \& Holdsworth 2019) included subtraction of median bias and flat-field correction using median-combined frames from exposures of the twilight sky. TEAPhot employs the method of adaptive elliptical aperture photometry, which was used to create calibrated science images and a differential light curve, using the bright $(\mathrm{V}=12.1)$ reference star, UCAC2 20781392. The differential light curve is shown in Fig. 13.

The light curve shows strong flickering, typical of CVs, and reminiscent of some intermediate polars which exhibit spinrelated periodic modulations. We therefore subjected the light curve to a Lomb-Scargle period analysis, probing frequencies as high as the Nyquist limit (0.6 s). Except for a strong peak at $0.480 \mathrm{mHz}$, there were no other significant peaks. The result is shown in Fig. 14. The strong peak corresponds to a period of $2080 \pm 100 \mathrm{~s}$ (34.7 min).

\section{Results and discussion}

We have analysed space- and ground-based data of SRGt 062340.2-265715, an object initially detected as an X-ray transient with the two X-ray instruments onboard SRG. Follow-up data, in particular the initial spectroscopy, uniquely identify the object as a cataclysmic variable thus confirming Denisenko's previous classification. At $G=12.5 \mathrm{mag}$ the object is clearly among the brighter examples of its class. An important question is to which, if any, CV-family the object belongs.

The spectra obtained and its location in the color-magnitude diagram (see Fig. 15) all strongly suggest that it belongs to the nova-like subclass. It is worth being mentioned that no dwarf 
nova outburst was recorded in the multi-year monitoring observations by CRTS.

The great majority of novalikes are found above the CV period gap. It is thus tempting to associate the 3.9 hour periodicity found in ASAS-SN and TESS data (sector 6) with the orbital period of the binary. The disappearance of this period in TESS sector 33 data is puzzling. Is it premature to associate the 3.9 hour periodicity with the orbital period or is the photometric phenomenology completely changed in the much higher accretion state of TESS sector 33 data? Time-resolved spectroscopy is needed to uniquely identify the orbital period by tracing both the absorption lines and the emission cores through an orbital cycle. The blue optical continuum and the absorption lines are thought to originate from an optically thick accretion disk. The narrow emission lines might be resolved in two components, one to be associated with the cooler outer parts of the disk and one originating from the irradiated hemisphere of the donor star (see e.g. Beuermann \& Thomas 1990; Hernandez et al. 2017, for templates), each one with its own radial velocity curve.

The production sites of X-rays in non-magnetic novalikes are. debated. Originally $\mathrm{X}$-rays were assumed to originate from the boundary layer (BL) between the disk and the white dwarf with a blackbody-like spectral shape but the predicted blackbodies were not found (Mauche \& Mukai 2002). The BL is nevertheless mostly regarded as the X-ray production site (Mukai 2017) but other locations like shocked circumstellar material, the polar caps, a central corona, a partially obscured boundary layer or advective hot flows are discussed (Zemko et al. 2014; Dobrotka et al. 2017; Balman 2020). The already observed high degree of X-ray variability on long and short time scales, i.e. betweeen eRASS surveys and between erodays, together with dedicated spectral observations may shed new light on this old question. If belonging to the non-magnetic novalikes, SRGt062340.2-265715 likely does not belong to the VY Scl subclass, because it does not show the typical anti-dwarf nova dimmings by $\sim 4$ mag.

Accretion in the intermediate polars may happen via streams and through disks. The disks may also be large and extend up to the Roche lobe of the accreting white dwarf (Hellier et al. 1991). The freely spinning white dwarf in an IP typically leads to very rich power spectra, in particular at X-ray wavelengths, with orbital, spin and different kinds of beat periods being traceable (dependent on the orbital inclination and the accretion mode, respectively, see e.g. Norton et al. 1996). Common to all IPs is their high degree of X-ray variability through self-eclipses and foreshortening of the accretion spots, and internal absorption by accretion curtains. The observed large X-ray variability within eRASS2 and between the two SRG surveys may thus argue for an IP classification. Also, the SAAO-discovered period of $35 \mathrm{~min}$ and the TESS discovered period at $25 \mathrm{~min}$ might support this view because such periods, if associated with the spin of the white dwarf are typical of IPs. In particular the TESSdiscovered periods at $25 \mathrm{~min}$ are suspicious of an IP nature because many IPs are observed to cluster around period ratios of $P_{\text {spin }} / P_{\text {orb }}=0.1$ (Norton et al. 2004, their figure 1) as implied for SRGt 062340.2-265715. To firmly classify the object as an IP, however, one needs the detection of a stable spin period, which is not found. The appearance and disappearance of periods at around half an hour is perhaps difficult to explain in either classification.

Optical power spectra of IPs are, however, affected by reprocessed radiation. Better insight is possible from a decent uninterrupted X-ray observation to uncover the intrinsic variability of the source on short timescales. The existing X-ray observations

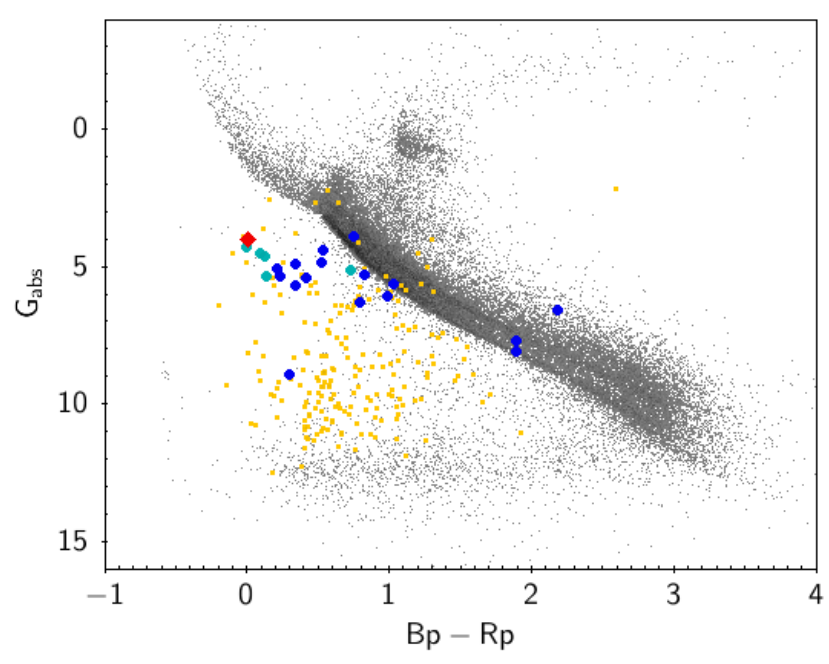

Fig. 15. Color magnitude diagram for eRASS2 sources. Each grey dot is an eRASS2 source that is matched (within $8 \operatorname{arcsec}$ ) with a Gaia source within $500 \mathrm{pc}$ (distances by Bailer-Jones et al. 2018). In dark yellow all CVs from the final Ritter \& Kolb catalogue, in green the nonmagnetic high-accretion rate UX UMas therein, in blue the Intermediate Polars from Mukai's compilation. The new CV SRGt 062340.2-265715 is identified by the red symbol.

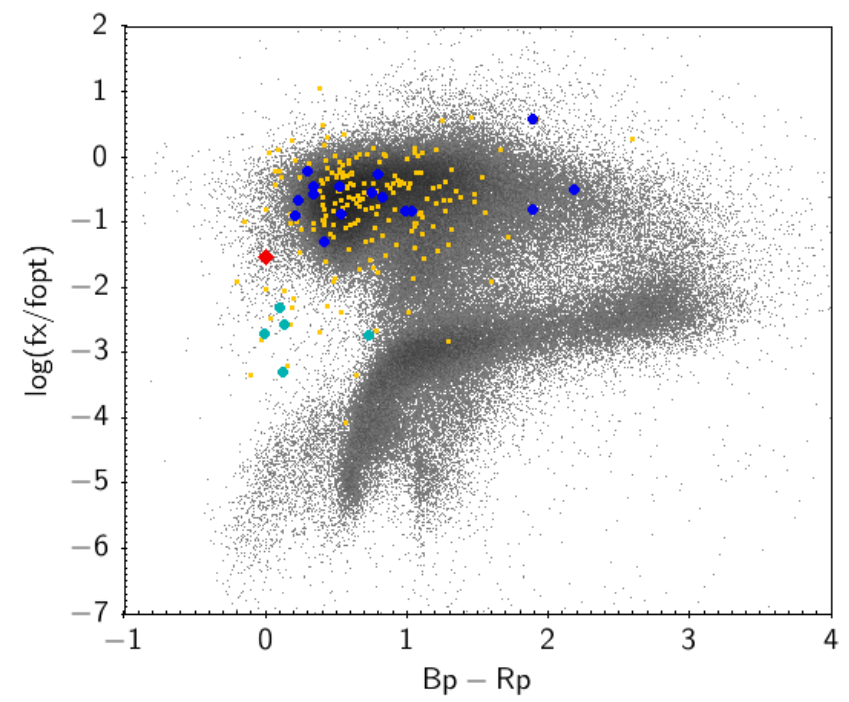

Fig. 16. X-ray to optical color-color diagram. Grey background objects are all eRASS2 sources matching within 8 arcsec with Gaia. Symbol colors are otherwise the same as in Fig. 15.

of SRGt 062340.2-265715 with SRG are insufficient to detect a $\sim 2000 \mathrm{~s}$ period. This will require an extensive future observation (e.g. with XMM-Newton).

The X-ray spectrum of SRGt 062340.2-265715 is not too strongly absorbed and can be modeled with a single purely thermal emission component. No separate soft X-ray component was found although the appearance of the Bowen blend seems to imply the existence of such component. The eRASS2 bolometric X-ray flux implies an accretion luminosity of $L_{\mathrm{X} \text {,bol }}=$ $4.8 \times 10^{32} \mathrm{erg} \mathrm{s}^{-1}$ which is at the low end for an IP and at the high end for an UX UMa system.

In addition to the color-magnitude diagram of Fig. 15 it is instructive to locate SRGt 062340.2-265715 in an X-ray to op- 
tical color-color diagram based on eROSITA and Gaia which is shown in Fig. 16. The diagrams were constructed in the following manner. The collaboration-internal preliminary eRASS2 catalogue was matched to within the 8-arcsec FHWM of the point spread functions of the telescope modules of eROSITA with Gaia DR2. For the matching objects, distances were determined following Bailer-Jones et al. (2021). We then used the final edition of the Ritter \& Kolb catalog (Ritter \& Kolb 2003) to locate the CVs. The optically bright nova-like CVs of the UX UMa type are identified with a different color in the diagrams. We also used the compilation of Koji Mukai ${ }^{4}$ to highlight the IPs that were identified in eRASS. The color $B p-R p$ is taken directly from Gaia data. The X-ray to optical color was computed as $\log \left(F_{\mathrm{X}}(0.6-2.3 \mathrm{keV})+\right.$ phot_g_mean_mag $/ 2.5+4.86$, the chosen energy band is the standard band 2 in the current version of the catalog pipeline (pipeline version 946 Brunner, H. et al. 2021, submitted to A\&A).

Our Fig. 15 is to be compared with Fig. 2 of Abril et al. (2020) which locates the CV sub-families in the Gaia-DR2 HRD. Whether the floor of objects in Fig. 15 at $G=13$ mag indicates real associations or is due to random associations needs to be seen. Most CVs are located between the white-dwarf sequence and the main sequence. They have X-ray luminosities that are orders of magnitude larger than the coronally active stars on the main sequence. The nova-like CVs, in particular the magnetic IPs, overlap in the color-magnitude diagram with coronally active stars but can be discerned through their much higher X-ray luminosity.

The color-color diagram of Fig. 16 has two main structures. The L-shaped sequence beginning at $(0.6,-5.5)$ with the knee at $(1,-3)$ delineates the active stars whereas the cloud around $(1,-0.5)$ is the domain of the AGN. Most of the CVs cannot be distinguished from the numerous AGN in such a diagnostic diagram as long as no astrometric information can be used for further discrimination of galactic and extragalactic objects (see also Comparat et al. 2020, their Fig. 11).

The transient CV SRGt 062340.2-265715 is located at very sparsely populated areas in both diagrams. It is optically among the most luminous X-ray detected CVs known. It is bluer than most non-magnetic UX UMa nova-like CVs and bluer than all IPs, although not too much separated from the extreme representatives of those two classes. On the other hand, its X-ray to optical color is between that of the UX UMas and the IPs. For an IP, SRGt 062340.2-265715 is X-ray underluminous by about $1 \mathrm{dex}$; for a non-magnetic novalike it is overluminous also by about 1 dex.

At Gaia magnitude of $12.5 \mathrm{mag}$, SRGt 062340.2-265715, if identified as an IP would by far be the IP with the brightest apparent magnitude in eRASS2 in the hemisphere with data rights for eROSITA_DE. The next brightest one is TV Col at phot_g_mean_mag $=13.9 \mathrm{mag}$. Just for comparison, the first soft X-ray discovered IP in the ROSAT all-sky survey, PQ Gem, was observed at phot_g_mean_mag $=14.1 \mathrm{mag}$.

Although only few objects are found in the vicinity of SRGt 062340.2-265715 in the diagnostic diagrams of Figs. 15 and 16 , several candidates are located around our new discovery that will be identified in the years to come via large-scale identification programs of serendipituous eROSITA sources with, e.g., the SDSS (Kollmeier et al. 2017). The journey has just begun.

Acknowledgements. We thank the referee, John Thorstensen, for constructive criticism. This work is based on data from eROSITA, the soft instrument aboard SRG, a joint Russian-German science mission supported by the Russian Space

\footnotetext{
$\overline{4}$ https://asd.gsfc.nasa.gov/Koji.Mukai/iphome/iphome.html
}

Agency (Roskosmos), in the interests of the Russian Academy of Sciences represented by its Space Research Institute (IKI), and the Deutsches Zentrum für Luftund Raumfahrt (DLR). The SRG spacecraft was built by Lavochkin Association (NPOL) and its subcontractors, and is operated by NPOL with support from the Max Planck Institute for Extraterrestrial Physics (MPE). The development and construction of the eROSITA X-ray instrument was led by MPE, with contributions from the Dr. Karl Remeis Observatory Bamberg \& ECAP (FAU ErlangenNuernberg), the University of Hamburg Observatory, the Leibniz Institute for Astrophysics Potsdam (AIP), and the Institute for Astronomy and Astrophysics of the University of Tübingen, with the support of DLR and the Max Planck Society. The Argelander Institute for Astronomy of the University of Bonn and the Ludwig Maximilians Universität Munich also participated in the science preparation for eROSITA. The eROSITA data shown here were processed using the eSASS/NRTA software system developed by the German eROSITA consortium. The Mikhail Pavlinsky ART-XC telescope is the hard X-ray instrument on board the $S R G$ observatory, a flagship astrophysical project of the Russian Federal Space Program realized by the Russian Space Agency, in the interests of the Russian Academy of Sciences. ART-XC was developed by the Space Research Institute (IKI, Moscow) and the Russian Federal Nuclear Center - All-Russian Scientific Research Institute for Experimental Physics (RFNC-VNIIEF, Sarov) with the participation of the NASA's Marshall Space Flight Center (MSFC). The ART-XC team thanks the Russian Space Agency, Russian Academy of Sciences and State Corporation Rosatom for the support of the $S R G$ project and ART-XC telescope. Some of the observations presented here were obtained with SALT under the transients followup programme 2018-2-LSP-001 (PI: DB), which is supported by Poland under grant no. MNiSW DIR/WK/2016/07. DB and LT also acknowledge research support from the National Research Foundation. We acknowledge the use of TESS High Level Science Products (HLSP) produced by the Quick-Look Pipeline (QLP) at the TESS Science Office at MIT, which are publicly available from the Mikulski Archive for Space Telescopes (MAST). Funding for the TESS mission is provided by NASA's Science Mission directorate. This work has made use of data from the Asteroid Terrestrial-impact Last Alert System (ATLAS) project. The Asteroid Terrestrial-impact Last Alert System (ATLAS) project is primarily funded to search for near earth asteroids through NASA grants NN12AR55G, 80NSSC18K0284, and 80NSSC18K1575; byproducts of the NEO search include images and catalogs from the survey area. This work was partially funded by Kepler/K2 grant J1944/80NSSC19K0112 and HST GO-15889, and STFC grants ST/T000198/1 and ST/S006109/1. The ATLAS science products have been made possible through the contributions of the University of Hawaii Institute for Astronomy, the Queen's University Belfast, the Space Telescope Science Institute, the South African Astronomical Observatory, and The Millennium Institute of Astrophysics (MAS), Chile. This paper includes data collected with the TESS mission, obtained from the MAST data archive at the Space Telescope Science Institute (STScI). Funding for the TESS mission is provided by the NASA Explorer Program. STScI is operated by the Association of Universities for Research in Astronomy, Inc., under NASA contract NAS 5-26555. MPM would like to thank Tom Maccarone for useful discussions.

\section{References}

Abril, J., Schmidtobreick, L., Ederoclite, A., \& López-Sanjuan, C. 2020, MNRAS, 492, L40

Astropy Collaboration, Price-Whelan, A. M., Sipőcz, B. M., et al. 2018, AJ, 156, 123

Bailer-Jones, C. A. L., Rybizki, J., Fouesneau, M., Demleitner, M., \& Andrae, R. 2021, AJ, 161, 147

Balman, S.. 2020, Advances in Space Research, 66, 1097

Baluev, R. V. 2008, MNRAS, 385, 1279

Beuermann, K. \& Thomas, H. C. 1990, A\&A, 230, 326

Bowman, D. M. \& Holdsworth, D. L. 2019, A\&A, 629, A21

Brunner, H., Liu, T., Lamer, G., et al. 2021, A\&A, submitted

Buckley, D. A. H., Swart, G. P., \& Meiring, J. G. 2006, in Proc. SPIE, Vol 6267 , Society of Photo-Optical Instrumentation Engineers (SPIE) Conference Series, $62670 \mathrm{Z}$

Childress, M., Vogt, F., Nielsen, J., \& Sharp, R. 2014, PyWiFeS: Wide Field Spectrograph data reduction pipeline

Comparat, J., Merloni, A., Dwelly, T., et al. 2020, A\&A, 636, A97

Coppejans, R., Gulbis, A. A. S., Kotze, M. M., et al. 2013, PASP, 125, 976

Cornelisse, R., Casares, J., Muñoz-Darias, T., et al. 2008, in American Institute of Physics Conference Series, Vol. 1010, A Population Explosion: The Nature \& Evolution of X-ray Binaries in Diverse Environments, ed. R. M. Bandyopadhyay, S. Wachter, D. Gelino, \& C. R. Gelino, 148-152

Crause, L. A., Sharples, R. M., Bramall, D. G., et al. 2014, in Society of PhotoOptical Instrumentation Engineers (SPIE) Conference Series, Vol. 9147, Proc. SPIE, 91476T

Crawford, S. M., Still, M., Schellart, P., et al. 2010, in Proc. SPIE, Vol. 7737, Observatory Operations: Strategies, Processes, and Systems III, 773725 
Czesla, S., Schröter, S., Schneider, C. P., et al. 2019, PyA: Python astronomyrelated packages

Dobrotka, A., Ness, J. U., Mineshige, S., \& Nucita, A. A. 2017, MNRAS, 468, 1183

Dopita, M., Rhee, J., Farage, C., et al. 2010, Ap\&SS, 327, 245

Drake, A. J., Djorgovski, S. G., Mahabal, A., et al. 2009, ApJ, 696, 870

Green, G. M., Schlafly, E., Zucker, C., Speagle, J. S., \& Finkbeiner, D. 2019, ApJ, 887, 93

Harlaftis, E. T. \& Horne, K. 1999, MNRAS, 305, 437

Heinze, A. N., Tonry, J. L., Denneau, L., et al. 2018, AJ, 156, 241

Hellier, C., Cropper, M., \& Mason, K. O. 1991, MNRAS, 248, 233

Hernandez, M. S., Zharikov, S., Neustroev, V., \& Tovmassian, G. 2017, MNRAS, 470, 1960

HI4PI Collaboration, Ben Bekhti, N., Flöer, L., et al. 2016, A\&A, 594, A116

Huang, C. X., Vanderburg, A., Pál, A., et al. 2020, Research Notes of the American Astronomical Society, 4, 204

Jenkins, J. M., Twicken, J. D., McCauliff, S., et al. 2016, in Society of PhotoOptical Instrumentation Engineers (SPIE) Conference Series, Vol. 9913, Software and Cyberinfrastructure for Astronomy IV, ed. G. Chiozzi \& J. C. Guzman, 99133E

Kniazev, A. Y., Gvaramadze, V. V., \& Berdnikov, L. N. 2016, MNRAS, 459, 3068

Kochanek, C. S., Shappee, B. J., Stanek, K. Z., et al. 2017, PASP, 129, 104502

Kollmeier, J. A., Zasowski, G., Rix, H.-W., et al. 2017, arXiv e-prints, arXiv: 1711.03234

Lightkurve Collaboration, Cardoso, J. V. d. M., Hedges, C., et al. 2018, Lightkurve: Kepler and TESS time series analysis in Python, Astrophysics Source Code Library

Lomb, N. R. 1976, Ap\&SS, 39, 447

Masci, F. J., Laher, R. R., Rusholme, B., et al. 2019, PASP, 131, 018003

Mauche, C. W. \& Mukai, K. 2002, ApJ, 566, L33

Mukai, K. 2017, PASP, 129, 062001

Norton, A. J., Beardmore, A. P., \& Taylor, P. 1996, MNRAS, 280, 937

Norton, A. J., Wynn, G. A., \& Somerscales, R. V. 2004, ApJ, 614, 349

Pavlinsky, M., Tkachenko, A., Levin, V., et al. 2021, arXiv e-prints, arXiv:2103.12479

Pichardo-Marcano, M. 2020, The Astronomer's Telegram, 14222, 1

Predehl, P., Andritschke, R., Arefiev, V., et al. 2021, A\&A, 647, A1

Ricker, G. R., Winn, J. N., Vanderspek, R., et al. 2014, in Society of PhotoOptical Instrumentation Engineers (SPIE) Conference Series, Vol. 9143 , Space Telescopes and Instrumentation 2014: Optical, Infrared, and Millimeter Wave, ed. J. Oschmann, Jacobus M., M. Clampin, G. G. Fazio, \& H. A. MacEwen, 914320

Ritter, H. \& Kolb, U. 2003, A\&A, 404, 301

Scargle, J. D. 1982, ApJ, 263, 835

Schachter, J., Filippenko, A. V., Kahn, S. M., \& Paerels, F. B. S. 1991, ApJ, 373, 633

Schwope, A. 2012, Mem. Soc. Astron. Italiana, 83, 844

Schwope, A., Semena, A., Maitra, C., et al. 2020, The Astronomer's Telegram, 14219,1

Shappee, B. J., Prieto, J. L., Grupe, D., et al. 2014, ApJ, 788, 48

Smith, J. C., Stumpe, M. C., Van Cleve, J. E., et al. 2012, PASP, 124, 1000

Stellingwerf, R. F. 1978, ApJ, 224, 953

Sunyaev, R., Arefiev, V., Babyshkin, V., et al. 2021, arXiv e-prints, arXiv:2104.13267

Tonry, J. L., Denneau, L., Heinze, A. N., et al. 2018, PASP, 130, 064505

Zemko, P., Orio, M., Mukai, K., \& Shugarov, S. 2014, MNRAS, 445, 869 